Document downloaded from:

http://hdl.handle.net/10251/176354

This paper must be cited as:

Ferrándiz-Catalá, B.; Denia, FD.; Martínez Casas, J.; Nadal, E.; Ródenas, JJ. (2020). Topology and shape optimization of dissipative and hybrid mufflers. Structural and Multidisciplinary Optimization. 62(1):269-284. https://doi.org/10.1007/s00158-020-02490-x

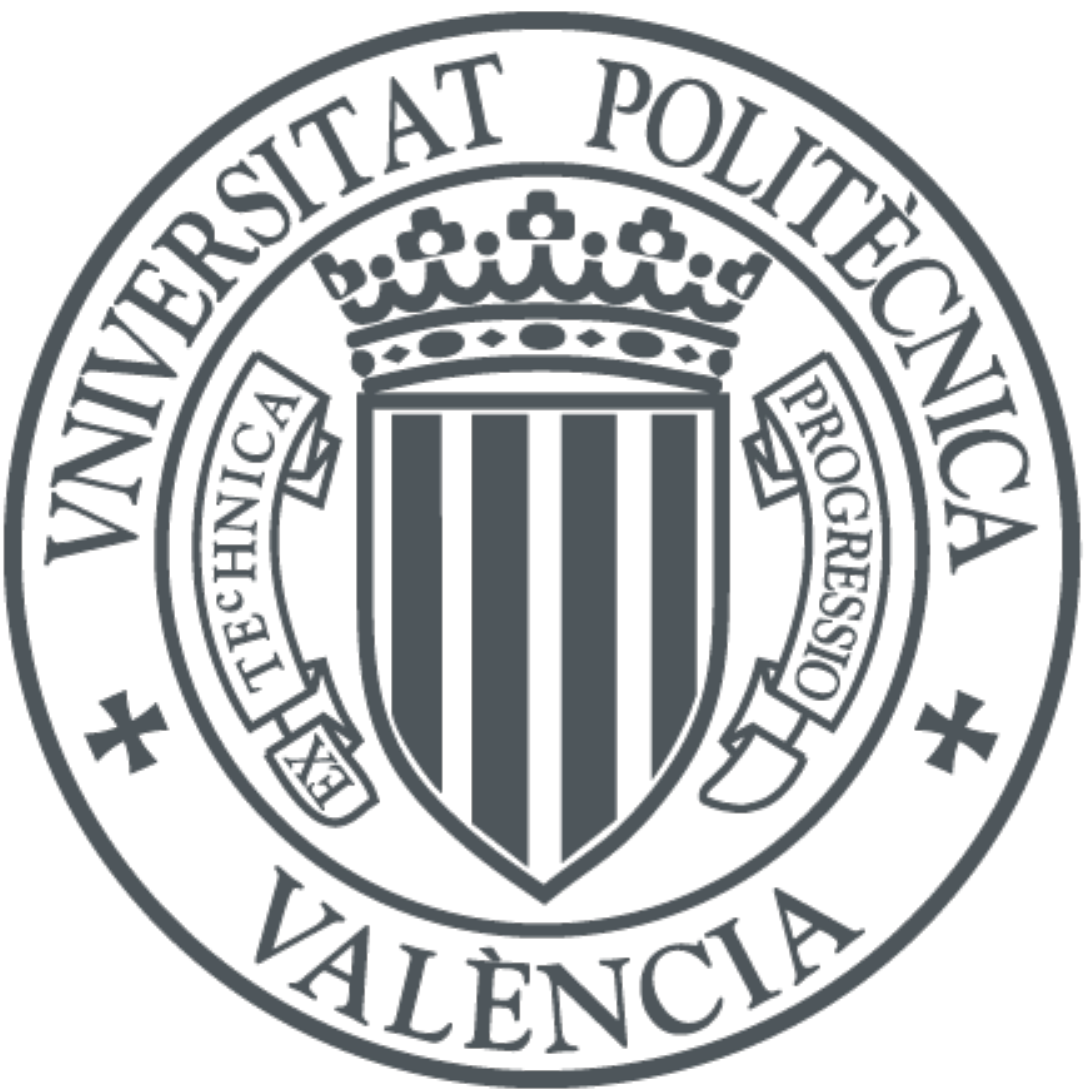

The final publication is available at

https://doi.org/10.1007/s00158-020-02490-x

Copyright Springer-Verlag

Additional Information 


\title{
Topology and shape optimization of dissipative and hybrid mufflers
}

\author{
B. Ferrándiz ${ }^{\mathrm{a}, *}$, F.D. Denia ${ }^{\mathrm{a}}$, J. Martínez-Casas ${ }^{\mathrm{a}}$, E. Nadal ${ }^{\mathrm{a}}$, J.J. Ródenas ${ }^{\mathrm{a}}$ \\ ${ }^{a}$ Centro de Investigación en Ingeniería Mecánica, Universitat Politècnica de València, \\ Camino de Vera s/n, 46022 Valencia, Spain
}

\begin{abstract}
This article presents a Topology Optimization (TO) method developed for maximizing the acoustic attenuation of a perforated dissipative muffler in the targeted frequency range by optimally distributing the absorbent material within the chamber. The Finite Element Method (FEM) is applied to the wave equation formulated in terms of acoustic pressure (chamber) and velocity potential (central duct, due to the existence of thermal gradients and mean flow) in order to evaluate the acoustic performance of the noise control device in terms of Transmission Loss (TL). Sound propagation through the chamber fibrous material is modelled considering complex equivalent acoustic properties, which vary spatially not only as a function of temperature, but also as a function of the filling density, since non-homogeneous density distributions are considered. The acoustic coupling at the perforated duct is performed by introducing a coordinate-dependent equivalent impedance. The objective function to maximize is expressed as the mean TL in the targeted frequency range. The sensitivities of this function with respect to the filling density of each element in the chamber are evaluated following the standard adjoint method. The Method of Moving Asymptotes (MMA) is used to update the design variables at each iteration of the TO process, keeping the weight of absorbent material equal or lower than a given value, while maximizing attenuation. Additionally, several particular designs inferred from the topology optimization results are analyzed. The sizing optimization of these rings is carried out simultaneously with the aforementioned TO process (density layout). A reactive chamber is added in order to evaluate the TL
\end{abstract}

\footnotetext{
* Corresponding author

Email address: borferca@upvnet.upv.es (B. Ferrándiz)
}

Preprint submitted to Structural and Multidisciplinary Optimization November 6, 2019 
of a hybrid muffler and its shape optimization is also carried out simultaneously with the aforementioned TO. Results show an increase in the muffler's mean TL at target frequencies, for all cases under study, while the amount of absorbent material used is maintained or even reduced.

Keywords: Silencer, Acoustic attenuation, Temperature gradient, Topology Optimization, Transmission Loss, Absorbent Material

\section{Introduction}

A large number of different muffler configurations is used nowadays; designs range from a simple expansion chamber connected to the inlet and outlet ducts, to intricate mufflers with several components combined together. Generally speaking, mufflers can be divided into reactive and dissipative configurations, depending on the acoustic phenomena that occur inside the device. Whereas reactive configurations cause the acoustic energy to be reflected back to the source, dissipative components, i.e., those containing absorbent material, convert the acoustic energy into heat [1].

Reactive mufflers can provide good acoustic attenuation at low to mid frequencies, even with relatively reduced dimensions [1], and their acoustic behaviour can be improved, at target frequencies, by applying sizing optimization to the components of the muffler, e.g., expansion chamber, extended ducts, resonators, etc. Barbieri and Barbieri [2] found an optimized geometry of a circular reactive chamber with extended inlet and outlet ducts by employing the Zoutendijk's feasible directions method [3]. Chang and Chiu [4] performed sizing optimization to designs with perforated duct using genetic algorithms (GA). GA were also used by De Lima et al. [5] in order to conduct the parametric optimization (sizing) of a reactive muffler with extended ducts, as well as the shape optimization of the duct's profile by the use of control points to approximate its boundary with cubic curves.

Topology optimization (TO) methods [6] can also be applied to reactive mufflers in order to distribute rigid-wall elements and create partitions inside the reactive chamber. The density approach is applied by employing material interpolation schemes, such as the Rational Approximation of Material Properties (RAMP) [7] in order to make the acoustic properties associated with each element of the FE mesh to vary from those of the air to those of soundhard material $[8,9]$. The sensitivities of the objective function with respect to the element densities are normally filtered [10] in order to avoid numeri- 
cal instabilities and mesh-dependent solutions. Lee [11] used the Method of Moving Asymptotes (MMA) [12] to create partitions in a reactive muffler and to increase the TL at targeted frequencies, experimentally validating the results. Lee and Kim [8] used the RAMP model along with the MMA to create partitions in an expansion chamber. Yedeg et al. [9] also used the MMA to create internal walls within a perforated muffler, obtaining components that resembled cylindrical pipes and Helmholtz resonators. On the other hand, Azevedo et al. [13] used the discrete optimization approach by employing Bidirectional Evolutionary Structural Optimization (BESO) method to create barriers within an expansion chamber using discrete variables.

Regarding dissipative mufflers, i.e., those containing absorbent material, they offer broadband attenuation essentially at mid and high frequencies [1]. Yoon [14] and Lee et al. [15] used the MMA to distribute elastic and poro-elastic material elements (as well as air elements) within the muffler's reactive chamber by the use of a thee-phase material scheme. Furthermore, Selamet et al. [16, 17] studied the acoustic behaviour of dissipative mufflers containing several layers of material with different compaction densities.

Considerable attenuation can be obtained in a broad range of the spectrum by using the so-called hybrid muffler, that combines reactive and dissipative chambers [18]. Chiu [19] used GA to maximize the attenuation of a hybrid muffler in a targeted frequency range, by optimizing the dimensions and the amount of absorbent material within the dissipative chamber (hence its filling density).

Moreover, the effect of temperature and thermal gradients on the behaviour of dissipative mufflers has been previously studied [20]. The FE model used in this study, which is fully explained in [20] and will be recalled in Section 2, is formulated in terms of acoustic velocity potential along the central duct, due to the existence of an axial flow and a heterogeneous medium; while pressure formulation is used at the chamber domain, where there is no mean flow.

This paper presents a method for improving the acoustic attenuation at target frequencies in dissipative and hybrid mufflers, through a TO approach that yields non-homogeneous absorbent material distributions. For the dissipative muffler, each finite element within the dissipative chamber is assigned a different design variable (filling density of the absorbent material within the element). Hence, the number of design variables coincides with the number of elements inside the chamber. As a consequence, the acoustic properties of the fibrous medium vary into the chamber. Regarding the hybrid muffler, 
the geometrical parameters of both dissipative and reactive chambers are also considered variables of the optimization process.

This paper is organized as follows: after this introduction, Section 2 provides the equations used to characterize the acoustic behaviour of dissipative and hybrid mufflers, considering thermal gradients and mean flow. The fibrous material in the dissipative chamber is modelled as an equivalent fluid, whose acoustic properties are evaluated within each element as a function of both its filling density and temperature, according to the models provided in Section 2.1 .

The high number of variables obtained makes the use of gradient-based algorithms a suitable method for the optimization problem presented in Section 3. The MMA [12] is utilized to update the filling densities of each element within the chamber, while maintaining or even reducing the amount of fibrous material used. The standard adjoint method recalled in Section 4 is considered to speed up the sensitivity analysis.

Numerical results are presented in Section 5. First the optimized filling density distribution is obtained for different working conditions. Next, some easy-to-fabricate models are obtained from the TO results and analysed. Additionally, a model with a dissipative chamber filled with rings of absorbent material, and a reactive chamber is considered. The filling density of each ring of material into the dissipative chamber, and the geometry of both chambers are optimized. Finally, Section 6 shows the main conclusions of this work.

\section{Acoustic problem}

Figure 1 shows a sketch of the perforated dissipative muffler considered in this section. It consists of a central air passage carrying a mean flow, and a surrounding annular chamber filled with absorbent material. Additionally, the perforated surface between the chamber and the central duct allows for acoustic interaction between them, while reducing back-pressure and preventing the absorbent material from being dragged out by the exhaust flow [16-18, 20-22].

The noise control device is divided into two subdomains: the central passage, denoted by $\Omega_{a}$, and the annular dissipative chamber, $\Omega_{m} . \Gamma_{p}$ denotes the perforated surface between them, $\Gamma_{a}$ and $\Gamma_{m}$ represent the rigid boundaries of the muffler (with normal velocity $U_{n}$ equal to zero), while $\Gamma_{i n}$ and $\Gamma_{\text {out }}$ stand for the inlet and outlet sections of the muffler. 


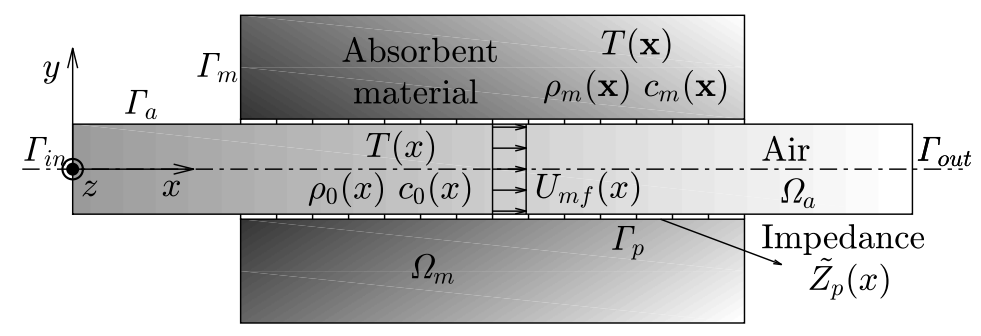

Figure 1: Perforated dissipative muffler with heterogeneous absorbent material.

Following the approach presented in [20], an axial temperature gradient is considered at the central passage, which causes not only the acoustic properties of the air (density $\rho_{0}$ and speed of sound $c_{0}$ ) to vary with respect to the $x$ coordinate, but also the mean flow speed $U_{m f}$. On the other hand, axial and radial temperature variations are considered inside the chamber. The fibrous medium can be modelled as an equivalent fluid, in terms of its position-dependent complex equivalent properties, $\rho_{m}(\mathbf{x})$ and $c_{m}(\mathbf{x})$, which can be expressed, for a given material, as a function of filling density $\rho_{b}(\mathbf{x})$, temperature $T(\mathbf{x})$ and acoustic frequency $f$. Finally, the perforated duct is modelled by evaluating the acoustic impedance $\tilde{Z}_{p}(x)$.

\subsection{Material characterization}

Absorbent materials can be modelled as equivalent fluids by using the corresponding complex acoustic properties [23]. Delany and Bazley's empirical model [24] is used in this study to estimate the characteristic acoustic impedance $Z_{m}$ and material wavenumber $k_{m}$ (or equivalenty the propagation constant) in terms of $f$ and steady airflow resistivity $R$.

On the other hand, $R$ can be evaluated at room temperature as a function of the filling density $\rho_{b}$ by the expression [25]

$$
R_{0}=A_{1} \rho_{b}^{A_{2}}
$$

where coefficients $A_{1}=1.0831$ and $A_{2}=1.8279$ are obtained from experimental data for the material under study, Owens Corning texturized fibreglass roving [18], with $R_{0}=4896 \mathrm{~Pa} \mathrm{~s} / \mathrm{m}^{2}$ for $\rho_{b}=100 \mathrm{~kg} / \mathrm{m}^{3}$, and $R_{0}=17378 \mathrm{~Pa} \mathrm{~s} / \mathrm{m}^{2}$ for $\rho_{b}=200 \mathrm{~kg} / \mathrm{m}^{3}$ [16]. This interpolation is hereinafter considered valid for $\rho_{b}$ between $50 \mathrm{~kg} / \mathrm{m}^{3}$ and $250 \mathrm{~kg} / \mathrm{m}^{3}$ [16-18], and any value of filling density between these two limits is considered suitable from a manufacturing point of view. 
Additionally, Christie's power law [26] is used in this study to evaluate $R$ at high temperature

$$
R(\mathbf{x})=R_{0}(\mathbf{x})\left(\frac{T(\mathbf{x})+273.15}{T_{0}+273.15}\right)^{0.6}
$$

with $T_{0}=25^{\circ} \mathrm{C}$. Once $R(\mathbf{x})$ is known, Delany and Bazley's aforementioned expressions for $Z_{m}(\mathbf{x})$ and $k_{m}(\mathbf{x})$ are [24]

$$
\begin{aligned}
Z_{m}(\mathbf{x}) & =Z_{0}(\mathbf{x})\left(1+a_{5} \xi(\mathbf{x})^{a_{6}}-j a_{7} \xi(\mathbf{x})^{a_{8}}\right) \\
k_{m}(\mathbf{x}) & =k_{0}(\mathbf{x})\left(1+a_{3} \xi(\mathbf{x})^{a_{4}}-j a_{1} \xi(\mathbf{x})^{a_{2}}\right)
\end{aligned}
$$

$Z_{0}(\mathbf{x})$ and $k_{0}(\mathbf{x})$ being the acoustic characteristic impedance of the air and its wavenumber, while the dimensionless frequency parameter is defined by $\xi(\mathbf{x})=\rho_{0}(\mathbf{x}) f / R(\mathbf{x})$. Coefficients and exponents $a_{i}$ for the material studied are shown in Table 1, and are kept constant with temperature [26-28]. Finally, the equivalent density $\rho_{m}(\mathbf{x})$ and speed of sound $c_{m}(\mathbf{x})$ are expressed as

$$
\begin{aligned}
& \rho_{m}(\mathbf{x})=\frac{Z_{m}(\mathbf{x})}{c_{m}(\mathbf{x})}, \\
& c_{m}(\mathbf{x})=\frac{\omega}{k_{m}(\mathbf{x})},
\end{aligned}
$$

$\omega$ being the angular frequency of the acoustic excitation.

\begin{tabular}{lr}
\hline \multicolumn{2}{l}{ Texturized fibre glass } \\
\hline$a_{1}$ & 0.189 \\
$a_{2}$ & -0.595 \\
$a_{3}$ & 0.160 \\
$a_{4}$ & -0.577 \\
$a_{5}$ & 0.095 \\
$a_{6}$ & -0.754 \\
$a_{7}$ & 0.085 \\
$a_{8}$ & -0.732 \\
\hline
\end{tabular}

Table 1: Coefficients and exponents for the calculation of the equivalent acoustic properties. 


\subsection{Hybrid FE formulation}

This section describes the FE formulation of the acoustic problem and the role played by the design variables (such as the filling density $\rho_{b}$ of each element or the chamber dimensions $L_{x}$ and $L_{r}$ ) in the equations. Additionally, the derivatives of the global system matrices with respect to the design variables are obtained in Appendix A.

The FE model described in [20] is used throughout this paper and recalled in this Section for the sake of convenience. The wave propagation model formulated in terms of acoustic velocity potential within $\Omega_{a}$, and in terms of acoustic pressure within $\Omega_{m}$ is explained in Sections 2.2.1 and 2.2.2, whereas the coupling between them across the perforated surface is recalled in Sections 2.2.3 and 2.2.4. The reader is referred to [20] for further details.

\subsubsection{Wave propagation along the central passage domain $\Omega_{a}$}

An axial temperature gradient is considered along the central passage. This implies the acoustic properties of the air $c_{0}(x)$ and $\rho_{0}(x)$ to vary axially within $\Omega_{a}$. In addition, a mean axial flow may be considered, with velocity $\mathbf{U}_{m f}(x)=\left\{U_{m f}(x), 0,0\right\}$. A convenient form of the wave equation for the described medium is the one formulated in terms of velocity potential $\phi[29]$ :

$$
\nabla \cdot\left(\rho_{0} \nabla \phi\right)-\rho_{0} \frac{\mathrm{D}}{\mathrm{D} t}\left(\frac{1}{c_{0}^{2}} \frac{\mathrm{D}}{\mathrm{D} t} \phi\right)=0 .
$$

Acoustic velocity $\mathbf{u}$ and pressure $p$ can be obtained from $\phi$ as [29]

$$
\begin{aligned}
& \mathbf{u}=\{u, v, w\}^{T}=\nabla \phi, \\
& p=-\rho_{0} \frac{\mathrm{D} \phi}{\mathrm{D} t} .
\end{aligned}
$$

A harmonic time dependence of the acoustic magnitudes involved is assumed, with $\phi(\mathbf{x}, t)=\Phi(\mathbf{x}) e^{j \omega t}$ and $p(\mathbf{x}, t)=P(\mathbf{x}) e^{j \omega t}$. Hence the total time derivative can be written as

$$
\frac{\mathrm{D}}{\mathrm{D} t}=\frac{\partial}{\partial t}+\mathbf{U}_{m f} \cdot \nabla=j \omega+U_{m f} \frac{\partial}{\partial x},
$$

$j$ being the imaginary unit. By introducing Eq. (10) into Eq. (7), it is 
obtained [20]

$$
\begin{aligned}
\nabla \cdot\left(\rho_{0} \nabla \Phi\right) & -\frac{\rho_{0} U_{m f}^{2}}{c_{0}^{2}} \frac{\partial^{2} \Phi}{\partial x^{2}}-\frac{2 \rho_{0} j \omega U_{m f}}{c_{0}^{2}} \frac{\partial \Phi}{\partial x}-\rho_{0} U_{m f}^{2} \frac{\partial\left(1 / c_{0}^{2}\right)}{\partial x} \frac{\partial \Phi}{\partial x} \\
& -\frac{\rho_{0} U_{m f}}{c_{0}^{2}} \frac{\partial U_{m f}}{\partial x} \frac{\partial \Phi}{\partial x}-\rho_{0} j \omega U_{m f} \frac{\partial\left(1 / c_{0}^{2}\right)}{\partial x} \Phi+\frac{\rho_{0} \omega^{2}}{c_{0}^{2}} \Phi=0
\end{aligned}
$$

The weighted residual statement (with weighting function $\Psi$ ) and the divergence theorem [30] are applied to Eq. (11). Further manipulation yields $[20]$

$$
\begin{gathered}
-\int_{\Omega_{a}} \rho_{0} \nabla^{T} \Psi \mathbf{M} \nabla \Phi \mathrm{d} \Omega \\
\quad+\int_{\Omega_{a}} \Psi\left(\frac{U_{m f}^{2}}{c_{0}^{2}} \frac{\partial \rho_{0}}{\partial x}+\frac{\rho_{0} U_{m f}}{c_{0}^{2}} \frac{\partial U_{m f}}{\partial x}-\frac{2 \rho_{0} j \omega U_{m f}}{c_{0}^{2}}\right) \frac{\partial \Phi}{\partial x} \mathrm{~d} \Omega \\
+\int_{\Omega_{a}} \Psi\left(-\rho_{0} j \omega U_{m f} \frac{\partial\left(1 / c_{0}^{2}\right)}{\partial x}+\frac{\rho_{0} \omega^{2}}{c_{0}^{2}}\right) \Phi \mathrm{d} \Omega+\int_{\Gamma_{a}} \rho_{0} \Psi \mathbf{n}^{T} \mathbf{M} \nabla \Phi \mathrm{d} \Gamma=0
\end{gathered}
$$

where $\mathbf{n}$ is the outward normal unit vector to the boundary $\Gamma$, and $\mathbf{M}$ is the matrix defined by

$$
\mathbf{M}=\left[\begin{array}{ccc}
1-M_{m f}^{2} & 0 & 0 \\
0 & 1 & 0 \\
0 & 0 & 1
\end{array}\right]
$$

$M_{m f}(x)=U_{m f}(x) / c_{0}(x)$ being the Mach number. The FE discretization given by Eqs. (14) and (15) is applied to Eq. (12), where $\mathbf{N}$ is the row vector of nodal shape functions, and $\tilde{\boldsymbol{\Phi}}$ and $\tilde{\boldsymbol{\Psi}}$ are the vectors containing the nodal values of $\Phi$ and $\Psi$.

$$
\begin{aligned}
& \Phi(\mathbf{x})=\mathbf{N}(\mathbf{x}) \tilde{\boldsymbol{\Phi}} \\
& \Psi(\mathbf{x})=\mathbf{N}(\mathbf{x}) \tilde{\boldsymbol{\Psi}}
\end{aligned}
$$

The use of the Galerkin approach [30] leads to the following algebraic system, expressed here in compact notation [20]:

$$
\left(\mathbf{K}_{a}+j \omega\left(\mathbf{C}_{a 1}+\mathbf{C}_{a 2}\right)-\omega^{2} \mathbf{M}_{a}\right) \tilde{\boldsymbol{\Phi}}=\mathbf{F}_{a}
$$


where the stiffness, damping and mass matrices, as well as the force vector are respectively [20]

$$
\begin{aligned}
\mathbf{K}_{a} & =\sum_{e=1}^{N_{a}^{e}} \int_{\Omega_{a}^{e}} \rho_{0}(\nabla \mathbf{N})^{T} \mathbf{M} \nabla \mathbf{N} \mathrm{d} \Omega \\
\mathbf{C}_{a 1} & =\sum_{e=1}^{N_{a}^{e}} \int_{\Omega_{a}^{e}} \frac{2 \rho_{0} U_{m f}}{c_{0}^{2}} \mathbf{N}^{T} \frac{\partial \mathbf{N}}{\partial x} \mathrm{~d} \Omega \\
\mathbf{C}_{a 2} & =\sum_{e=1}^{N_{a}^{e}} \int_{\Omega_{a}^{e}} \rho_{0} U_{m f} \frac{\partial\left(1 / c_{0}^{2}\right)}{\partial x} \mathbf{N}^{T} \mathbf{N} \mathrm{d} \Omega, \\
\mathbf{M}_{a} & =\sum_{e=1}^{N_{a}^{e}} \int_{\Omega_{a}^{e}} \frac{\rho_{0}}{c_{0}^{2}} \mathbf{N}^{T} \mathbf{N} \mathrm{d} \Omega \\
\mathbf{F}_{a} & =\sum_{e=1}^{N_{a}^{e}} \int_{\Gamma_{a}^{e} \cap \Gamma} \rho_{0} \mathbf{N}^{T} \mathbf{n}^{T} \mathbf{M} \nabla \Phi \mathrm{d} \Gamma \\
& =\sum_{e=1}^{N_{a}^{e}} \int_{\Gamma_{a}^{e} \cap \Gamma_{b c}} \rho_{0} \mathbf{N}^{T}\left(1-M_{m f}^{2}\right) \frac{\partial \Phi}{\partial n}+\int_{\Gamma_{a}^{e} \cap \Gamma_{p}} \rho_{0} \mathbf{N}^{T} \frac{\partial \Phi}{\partial n} \mathrm{~d} \Gamma,
\end{aligned}
$$

$N_{a}^{e}, \Omega_{a}^{e}$ and $\Gamma_{a}^{e}$ being the number of elements within $\Omega_{a}$, as well as the domain and boundary of each of these elements in the summation term. A normal acoustic velocity to $\Gamma$ may exist at those boundaries where the natural rigid wall condition is not satisfied [31], i.e., $\Gamma_{b c}=\Gamma_{\text {in }} \cup \Gamma_{\text {out }}$ (inlet/outlet sections) and $\Gamma_{p}$ (perforated duct surface).

\subsubsection{Wave propagation in the dissipative chamber}

In the absence of mean flow, and considering a heterogeneous medium, the simpler harmonic form of the wave equation expressed in terms of pressure can be used [20-22, 29]

$$
\nabla \cdot\left(\frac{1}{\rho_{m}} \nabla P\right)+\frac{\omega^{2}}{\rho_{m} c_{m}^{2}} P=0 .
$$

The FE method is applied to Eq. (22) in an analogous way to Section 2.2.1, yielding the following algebraic system, expressed here in compact form $[20]$

$$
\left(\mathbf{K}_{m}-\omega^{2} \mathbf{M}_{m}\right) \tilde{\mathbf{P}}=\mathbf{F}_{m},
$$


where the matrices $\mathbf{K}_{m}, \mathbf{M}_{m}$ and the vector $\mathbf{F}_{m}$ are defined below [20]

$$
\begin{aligned}
\mathbf{K}_{m} & =\sum_{e=1}^{N_{m}^{e}} \int_{\Omega_{m}^{e}} \frac{1}{\rho_{m}}(\nabla \mathbf{N})^{T}(\nabla \mathbf{N}) \mathrm{d} \Omega, \\
\mathbf{M}_{m} & =\sum_{e=1}^{N_{m}^{e}} \int_{\Omega_{m}^{e}} \frac{1}{\rho_{m} c_{m}^{2}} \mathbf{N}^{T} \mathbf{N} \mathrm{d} \Omega, \\
\mathbf{F}_{m} & =\sum_{e=1}^{N_{m}^{e}} \int_{\Gamma_{m}^{e} \cap \Gamma_{p}} \frac{1}{\rho_{m}} \mathbf{N}^{T} \frac{\partial P}{\partial n} \mathrm{~d} \Gamma,
\end{aligned}
$$

$N_{m}^{e}, \Omega_{m}^{e}$ and $\Gamma_{m}^{e}$ being the number of elements within $\Omega_{m}$, as well as the domain and boundary of each of these elements in the summation term. The equivalent acoustic properties of the poroelastic medium $\rho_{m}$ and $c_{m}$ are calculated according to Eqs. (5) and (6). Note that, although just one variable $\rho_{b}$ is assigned to each element, $\rho_{m}$ and $c_{m}$ might vary within $\Omega_{m}^{e}$ due to thermal effects.

\subsubsection{Acoustic coupling at the perforated duct}

The acoustic coupling between $\Omega_{a}$ and $\Omega_{m}$ is modelled by means of an impedance associated with the perforated surface. This is defined as the ratio of the acoustic pressure drop between both sides of the plate to the acoustic velocity across the orifices $U_{n}[1]$ :

$$
\tilde{Z}_{p}=\frac{P_{a}-P_{m}}{U_{n}},
$$

where $P_{a}$ and $P_{m}$ are the pressures at both sides of the perforated screen. On the other hand, recalling Eqs. (8), (9) and (10) within $\Omega_{a}$ yields

$$
P_{a}=-\rho_{0}\left(j \omega \Phi+U_{m f} \partial \Phi / \partial x\right) .
$$

Assuming continuity of normal velocity across the perforated surface [32], $U_{n_{a}}=-U_{n_{m}}$, and substituting $\partial \Phi / \partial n=U_{n}$ from Eq. (27) into the second term of Eq. (21), the contribution of the perforated surface to $\mathbf{F}_{a}$ is obtained $[20]$

$$
\mathbf{F}_{a}^{Z_{p}}=\int_{\Gamma_{a}^{e} \cap \Gamma_{p}} \rho_{0} \mathbf{N}^{T}\left(\frac{-\rho_{0}\left(j \omega \Phi+U_{m f} \partial \Phi / \partial x\right)}{\tilde{Z}_{p}}-\frac{P_{m}}{\tilde{Z}_{p}}\right) \mathrm{d} \Gamma .
$$


$\mathbf{F}_{m}^{Z_{p}}$ can be derived analogously, replacing the term $\partial P_{m} / \partial n=-\rho_{m} j \omega\left(-U_{n}\right)$ into Eq. (26), where $U_{n_{m}}$ is obtained from Eq. (27) [20].

$$
\mathbf{F}_{m}^{Z_{p}}=\int_{\Gamma_{a}^{e} \cap \Gamma_{p}} \mathbf{N}^{T}\left(\frac{\rho_{0} \omega^{2} \Phi-\rho_{0} j \omega U_{m f} \partial \Phi / \partial x}{\tilde{Z}_{p}}-\frac{j \omega P_{m}}{\tilde{Z}_{p}}\right) \mathrm{d} \Gamma
$$

No mesh continuity is required between both domains, although a conforming mesh has been used between $\Omega_{a}$ and $\Omega_{m}$ in order to simplify the computations, and shape functions at both sides of the perforated surface are therefore equal [20]. The force terms described in Eqs. (29) and (30) are moved to the left-hand side of the algebraic system, following the relations:

$$
\begin{aligned}
& \mathbf{F}_{a}^{Z_{p}}=\left(-\mathbf{K}_{a a}^{Z_{p}}-j \omega \mathbf{C}_{a a}^{Z_{p}}\right) \tilde{\boldsymbol{\Phi}}_{a}-\mathbf{K}_{a m}^{Z_{p}} \tilde{\mathbf{P}}_{m}, \\
& \mathbf{F}_{m}^{Z_{p}}=\left(-j \omega \mathbf{C}_{m a}^{Z_{p}}+\omega^{2} \mathbf{M}_{m a}^{Z_{p}}\right) \tilde{\boldsymbol{\Phi}}_{a}-j \omega \mathbf{C}_{m m}^{Z_{p}} \tilde{\mathbf{P}}_{m},
\end{aligned}
$$

obtaining the following new terms [20]

$$
\begin{aligned}
\mathbf{K}_{a a}^{Z_{p}} & =\sum_{e=1}^{N_{a}^{e}} \int_{\Gamma_{a}^{e} \cap \Gamma_{p}} \frac{\rho_{0}^{2} U_{m f} \mathbf{N}^{T}}{\tilde{Z}_{p}} \frac{\partial \mathbf{N}}{\partial x} \mathrm{~d} \Gamma \\
\mathbf{K}_{a m}^{Z_{p}} & =\sum_{e=1}^{N_{a}^{e}} \int_{\Gamma_{a}^{e} \cap \Gamma_{p}} \frac{\rho_{0} \mathbf{N}^{T} \mathbf{N}}{\tilde{Z}_{p}} \mathrm{~d} \Gamma \\
\mathbf{C}_{a a}^{Z_{p}} & =\sum_{e=1}^{N_{a}^{e}} \int_{\Gamma_{a}^{e} \cap \Gamma_{p}} \frac{\rho_{0}^{2} \mathbf{N}^{T} \mathbf{N}}{\tilde{Z}_{p}} \mathrm{~d} \Gamma \\
\mathbf{C}_{m m}^{Z_{p}}= & \sum_{e=1}^{N_{a}^{e}} \int_{\Gamma_{m}^{e} \cap \Gamma_{p}} \frac{\mathbf{N}^{T} \mathbf{N}}{\tilde{Z}_{p}} \mathrm{~d} \Gamma \\
\mathbf{C}_{m a}^{Z_{p}}= & \sum_{e=1}^{N_{a}^{e}} \int_{\Gamma_{m}^{e} \cap \Gamma_{p}} \frac{\rho_{0} U_{m f} \mathbf{N}^{T}}{\tilde{Z}_{p}} \frac{\partial \mathbf{N}}{\partial x} \mathrm{~d} \Gamma \\
\mathbf{M}_{m a}^{Z_{p}}= & \sum_{e=1}^{N_{a}^{e}} \int_{\Gamma_{m}^{e} \cap \Gamma_{p}} \frac{\rho_{0} \mathbf{N}^{T} \mathbf{N}}{\tilde{Z}_{p}} \mathrm{~d} \Gamma .
\end{aligned}
$$

\subsubsection{Perforated duct impedance models}

In order to model the acoustic coupling at a perforated circular duct separating an air passage with grazing mean flow and a chamber with no absorbent material, the empirical impedance model presented by Lee and Ih 
[33] has been found to show good correlation with experimental data [32] and is therefore used in this study. The dimensionless impedance for a perforated surface in the presence of grazing mean flow is

$$
\varsigma_{p}(x)=\frac{Z_{p}(x)}{\rho_{0}(x) c_{0}(x)}=\alpha(x)+j \beta(x) .
$$

The values of the real (resistance) and imaginary (reactance) parts depend on the geometric parameters of the perforated plate: its thickness $t_{p}$, porosity $\sigma$ and diameter of the orifices $d_{h}$, as well as $f$ and $M_{m f}$, and its computation is described in [20]. In order to capture the effect of the fibrous material, good correlation with experimental results is found for Kirby and Denia's expression shown below [32]

$$
\tilde{Z}_{p}(x)=Z_{0}(x)\left(\varsigma_{p}(x)+\frac{j 0.425 k_{0}(x) d_{h}\left(\rho_{m}(x) / \rho_{0}(x)-1\right) F(\sigma)}{\sigma}\right),
$$

where the hole interaction factor $F(\sigma)$ is approximated in this study by the mean value $[32,34]$ given by Ingard [35] and Fok [36] expressions, denoted below by $F_{I}$ and $F_{F}$, respectively

$$
\begin{aligned}
& F_{I}(\sigma)=1-0.7 \sqrt{\sigma}, \\
& F_{F}(\sigma)=1-1.41 \sqrt{\sigma}+0.34 \sqrt{\sigma^{3}}+0.34 \sqrt{\sigma^{5}} .
\end{aligned}
$$

\subsubsection{Boundary conditions at the outlet}

TL computations require an anechoic termination [1]. This can be achieved by prescribing an acoustic impedance at the outlet section equal to the characteristic impedance of the propagation medium:

$$
Z=\frac{P}{U_{n}}=\frac{P}{\partial \Phi / \partial x}=Z_{0}=\rho_{0} c_{0}
$$

Introducing $P$ from Eq. (43) into Eq. (28), and solving for $\partial \Phi / \partial x$ leads to $[20]$

$$
\frac{\partial \Phi}{\partial x}=-\frac{j \omega \rho_{0} \Phi}{Z_{0}+\rho_{0} U_{m f}} .
$$

Eq. (44) is then introduced into the first term of Eq. (21), and the resultant expression is evaluated at the outlet section, obtaining

$$
\mathbf{F}_{a}^{Z_{\text {out }}}=-j \omega \rho_{0}^{2} \frac{1-M_{m f}^{2}}{Z+\rho_{0} U_{m f}} \sum_{e=1}^{N_{e}^{\text {out }}} \int_{\Gamma_{e} \cap \Gamma_{\text {out }}} \mathbf{N}^{T} \Phi \mathrm{d} \Gamma .
$$


Eq. (45) is moved to the left-hand side of Eq. (16). In compact form, $\mathbf{F}_{a}^{Z_{\text {out }}}=-j \omega \mathbf{C}_{a}^{Z_{\text {out }}} \tilde{\boldsymbol{\Phi}}_{a}$, with the new matrix $\mathbf{C}_{a}^{Z_{\text {out }}}$ given by:

$$
\mathbf{C}_{a}^{Z_{\text {out }}}=\rho_{0}^{2} \frac{1-M_{m f}^{2}}{Z+\rho_{0} U_{m f}} \sum_{e=1}^{N_{e}^{\text {out }}} \int_{\Gamma_{e} \cap \Gamma_{\text {out }}} \mathbf{N}^{T} \mathbf{N} \mathrm{d} \Gamma .
$$

\subsubsection{Final system of equations}

The resulting global system of equations is:

$$
\begin{aligned}
\left(\left[\begin{array}{cc}
\mathbf{K}_{a}+\mathbf{K}_{a a}^{Z_{p}} & \mathbf{K}_{a m}^{Z_{p}} \\
0 & \mathbf{K}_{m}
\end{array}\right]\right. & +j \omega\left[\begin{array}{cc}
\mathbf{C}_{a 1}+\mathbf{C}_{a 2}+\mathbf{C}_{a}^{Z_{o u t}}+\mathbf{C}_{a a}^{Z_{p}} & 0 \\
\mathbf{C}_{m a}^{Z_{p}} & \mathbf{C}_{m m}^{Z_{p}}
\end{array}\right] \\
& \left.-\omega^{2}\left[\begin{array}{cc}
\mathbf{M}_{a} & 0 \\
\mathbf{M}_{m a}^{Z_{p}} & \mathbf{M}_{m}
\end{array}\right]\right)\left\{\begin{array}{c}
\tilde{\boldsymbol{\Phi}}_{a} \\
\tilde{\mathbf{P}}_{m}
\end{array}\right\}=\left\{\begin{array}{c}
\mathbf{F}_{a}^{i n} \\
0
\end{array}\right\} .
\end{aligned}
$$

However, in the present study excitation at the inlet section is modelled by means of Dirichlet boundary conditions, with $\Phi=1$ on $\Gamma_{i n}$ for simplicity. A suitable modification of rows and columns in Eq. (47) is then required, following [37]. Additionally, $\mathbf{F}_{a}^{i n}=\mathbf{0}$. Finally, the value of the objective function is obtained using the solution of the acoustic field within the silencer, as shown in Section 2.3.

\subsection{Objective Function. Transmission Loss}

The acoustic attenuation of a muffler can be measured in terms of Transmission Loss (TL), which is defined as the ratio of the incident power on the noise control device to the one transmitted through the outlet section (with anechoic conditions). It can be expressed as [1]

$$
T L=10 \log \left(\frac{\left|P_{\text {in }}^{+}\right|^{2} S_{\text {in }}\left(1+M_{\text {in }}^{2}\right) /\left(2 \rho_{\text {in }} c_{\text {in }}\right)}{\left|P_{\text {out }}^{+}\right|^{2} S_{\text {out }}\left(1+M_{\text {out }}^{2}\right) /\left(2 \rho_{\text {out }} c_{\text {out }}\right)}\right),
$$

where $P^{+}, S$ and $M$ denote the amplitude of the progressive wave, the crosssection area of the tube and the Mach number, respectively (subscripts in and out denote the inlet and outlet sections). By assuming that the inlet and outlet ducts are long enough to avoid three-dimensional effects at $\Gamma_{\text {in }}$ and $\Gamma_{\text {out }}$ (generated at the expansion and contraction of the muffler chamber), plane wave propagation is guaranteed for the frequencies analyzed (up to 3200 $\mathrm{Hz})$, and the progressive wave at the inlet section is $P_{i n}^{+}=\left(P_{i n}+Z_{0} U_{i n}\right) / 2$, 
whereas the lack of regressive wave at the outlet tube leads to $P_{\text {out }}^{+}=P_{\text {out }}$ [22]. Given Eqs. (8), (9) and (10), it leads to

$$
\begin{aligned}
P_{\text {in }}^{+} & =\frac{1}{2}\left(-\rho_{\text {in }}\left(j \omega \tilde{\boldsymbol{\Phi}}\left(n_{1}^{\text {in }}\right)+U_{m f}^{\text {in }} \frac{\partial \Phi}{\partial x}\left(n_{1}^{\text {in }}\right)\right)+Z_{0} \frac{\partial \Phi}{\partial x}\left(n_{1}^{\text {in }}\right)\right), \\
P_{\text {out }}^{+} & =-\rho_{\text {out }}\left(j \omega \tilde{\boldsymbol{\Phi}}_{\text {out }}\left(n_{3}^{\text {out }}\right)+U_{m f}^{\text {out }} \frac{\partial \Phi}{\partial x}\left(n_{3}^{\text {out }}\right)\right),
\end{aligned}
$$

where $n_{1}^{\text {in }}$ and $n_{3}^{\text {out }}$ are the central nodes of $\Gamma_{\text {in }}$ and $\Gamma_{\text {out }}$, respectively. The computation of $\partial \Phi / \partial x$ at $n_{1}^{\text {in }}$ and $n_{3}^{\text {out }}$ can be carried out, for example, through the nodal solution of $\Phi$ at additional nodes $n_{2}^{\text {in }}, n_{3}^{\text {in }}, n_{1}^{\text {out }}$, and $n_{2}^{\text {out }}$ within the corresponding elements (see Figure 2).

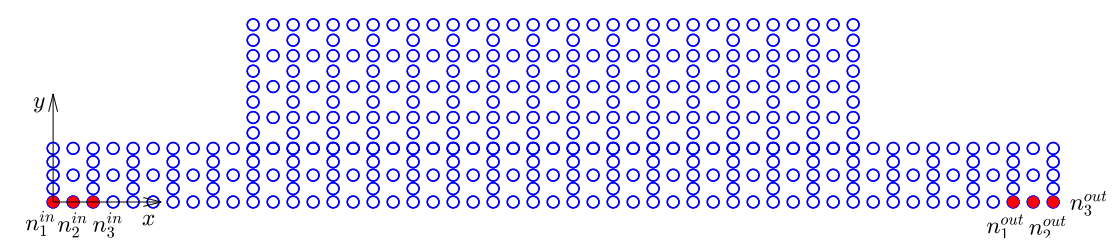

Figure 2: Nodes of the finite element mesh of a dissipative muffler.

\section{Optimization problem}

The optimization problem of designing a muffler targeted to operate in the frequency range $\left[\omega_{0}, \omega_{1}\right]$ can be stated as

$$
\begin{gathered}
\max _{\boldsymbol{v}} \quad f_{0}(\tilde{\boldsymbol{\Phi}}(\boldsymbol{v}))=\frac{1}{\omega_{1}-\omega_{0}} \int_{\omega_{0}}^{\omega_{1}} T L(\tilde{\boldsymbol{\Phi}}(\boldsymbol{v}), \omega) \mathrm{d} \omega, \\
\text { subject to } \quad g_{i}(\boldsymbol{v}) \leq 0 \quad \text { for } i=1, \ldots, m, \\
v_{j}^{\min } \leq v_{j} \leq v_{j}^{\max } \quad \text { for } j=1, \ldots, n,
\end{gathered}
$$

i.e., optimizing a set of $n$ design variables $v_{j}$, in order to maximize the mean TL in the targeted frequency range, while satisfying the $m$ constraints $g_{i}(\boldsymbol{v})$. Note that $\boldsymbol{v}$ is a general representation of the design variables. In general, $\boldsymbol{v}$ contains the bulk densities corresponding to the elements of the chamber. In Section 5.3 it will contain some additional variables such as the dimensions of the dissipative and reactive chambers. A weight constraint is included to limit the maximum amount of fibre, and to show the versatility of the proposed method to add constraints:

$$
g_{1}(\boldsymbol{v})=W(\boldsymbol{v})-W_{0} \leq 0
$$


$W_{0}$ being the maximally allowed weight. Simpson's numerical integration rule [38] with frequency spacing $\Delta \omega$ of $5 \mathrm{~Hz}$ is used in this study to approximate the integral in (51). As mentioned in Section 1, the optimization problem is solved at each iteration by the use of the gradient-based MMA [12] due to the high number of design variables. No filtering of sensitivities is performed.

Opposite to standard TO approaches, which look for material-void designs, the proposed method also allows for intermediate values of filling density, since obtaining areas with these filling density values is possible from a manufacturing point of view. Hence intermediate values of $\rho_{b}$ are not penalized.

Given a design point $\boldsymbol{v}$, an approximating subproblem is generated, in which the objective function is replaced by certain convex functions, using gradient information. The algorithm to solve this subproblem is beyond the scope of this paper and the reader is referred to [12] for its description. However, it has not been proven that the problem is convex, hence it is not possible to state that the solution reached after meeting the stopping criterion is the optimal design. The computation of the sensitivities of $f_{0}$ with respect to a perturbation of the design variables can be sped up (with respect to the finite difference method) by using the standard adjoint method [39], recalled in Section 4.

\section{Sensitivity analysis}

The standard adjoint method [39] is described below. The global algebraic system (47) is recalled here in compact form

$$
\boldsymbol{\kappa}(\boldsymbol{v}, \omega) \tilde{\boldsymbol{\Theta}}(\boldsymbol{v}, \omega)=\mathbf{F}
$$

where $\boldsymbol{\kappa}=\mathbf{K}+j \omega \mathbf{C}-\omega^{2} \mathbf{M}$ and the column vector $\tilde{\boldsymbol{\Theta}}$ contains the nodal solution in terms of acoustic pressure and velocity potential. As explained in Section 5, the force term $\mathbf{F}$ only includes the force terms derived from the excitation at the inlet, and does not depend on $\boldsymbol{v}$. At each iteration, for a certain frequency $\omega$, the augmented objective function is defined as follows:

$\widehat{f}_{0}(\tilde{\boldsymbol{\Theta}}(\boldsymbol{v}))=f_{0}(\tilde{\boldsymbol{\Theta}}(\boldsymbol{v}))-\boldsymbol{\lambda}^{T}(\boldsymbol{v})(\boldsymbol{\kappa}(\boldsymbol{v}) \tilde{\boldsymbol{\Theta}}(\boldsymbol{v})-\mathbf{F})-\overline{\boldsymbol{\lambda}}^{T}(\boldsymbol{v})(\overline{\boldsymbol{\kappa}(\boldsymbol{v}) \tilde{\boldsymbol{\Theta}}(\boldsymbol{v})-\mathbf{F}})$

$\boldsymbol{\lambda}(\boldsymbol{v})$ being the Lagrange multipliers column vector, and $\overline{(\cdot)}$ being the complex conjugate of $(\cdot)$. Differentiating Eq. (55) with respect to the design variable 
$v_{j}$ and reordering the terms yields

$$
\begin{aligned}
\frac{\mathrm{D} \widehat{f}_{0}}{\mathrm{D} v_{j}}=\frac{\partial f_{0}}{\partial v_{j}} & +\left(\frac{\partial \tilde{\boldsymbol{\Theta}}_{r}}{\partial v_{j}}\right)^{T}\left(-\boldsymbol{\kappa}^{T} \boldsymbol{\lambda}-\overline{\boldsymbol{\kappa}^{T} \boldsymbol{\lambda}}+\frac{\partial f_{0}}{\partial \tilde{\boldsymbol{\Theta}}_{r}}\right) \\
& +\left(\frac{\partial \tilde{\boldsymbol{\Theta}}_{i}}{\partial v_{j}}\right)^{T}\left(-j \boldsymbol{\kappa}^{T} \boldsymbol{\lambda}+j \overline{\boldsymbol{\kappa}^{T} \boldsymbol{\lambda}}+\frac{\partial f_{0}}{\partial \tilde{\boldsymbol{\Theta}}_{i}}\right) \\
& -\boldsymbol{\lambda}^{T}\left(\frac{\partial \boldsymbol{\kappa}}{\partial v_{j}} \tilde{\boldsymbol{\Theta}}\right)-\overline{\boldsymbol{\lambda}}^{T}\left(\overline{\frac{\partial \boldsymbol{\kappa}}{\partial v_{j}} \tilde{\boldsymbol{\Theta}}}\right)
\end{aligned}
$$

where the computation of the term $\partial \kappa / \partial v_{j}$ is explained in Appendix A.1 (derivative with respect to the filling density), Appendix A.2 (longitudinal dimension of the chamber) and Appendix A.3 (radial dimension). Column vectors $\partial f_{0} / \partial \tilde{\boldsymbol{\Theta}}_{r}$ and $\partial f_{0} / \partial \tilde{\boldsymbol{\Theta}}_{i}$ contain the derivatives of $f_{0}$ with respect to the real and imaginary parts of the nodal solution of the six nodes highlighted in Figure 2, and can be derived analytically from Eqs. (48), (49) and (50). In addition, $\partial f_{0} / \partial v_{j}=0$.

As the explicit form of the terms $\partial \tilde{\boldsymbol{\Theta}}_{r} / \partial v_{j}$ and $\partial \tilde{\boldsymbol{\Theta}}_{i} / \partial v_{j}$ is difficult to obtain, $\boldsymbol{\lambda}(\mathbf{x})$ is chosen so that

$$
\begin{gathered}
-\boldsymbol{\kappa}^{T} \boldsymbol{\lambda}-\overline{\boldsymbol{\kappa}^{T} \boldsymbol{\lambda}}+\frac{\partial f_{0}}{\partial \tilde{\boldsymbol{\Theta}}_{r}}=0 \\
-j \boldsymbol{\kappa}^{T} \boldsymbol{\lambda}+j \overline{\boldsymbol{\kappa}^{T} \boldsymbol{\lambda}}+\frac{\partial f_{0}}{\partial \tilde{\boldsymbol{\Theta}}_{i}}=0 .
\end{gathered}
$$

Multiplying Eq. (58) by $-j$ and adding Eq. (57) leads to [39]

$$
\boldsymbol{\lambda}=\frac{1}{2}\left(\boldsymbol{\kappa}^{T}(\mathbf{x})\right)^{-1}\left(\frac{\partial f_{0}}{\partial \tilde{\boldsymbol{\Theta}}_{r}}-j \frac{\partial f_{0}}{\partial \tilde{\boldsymbol{\Theta}}_{i}}\right) .
$$

The derivatives of the system matrices with respect to each design variable are obtained analytically as explained in Appendix A, and the sensitivity of the objective function with respect to each design variable $v_{j}$ is calculated as [39]

$$
\frac{\mathrm{D} \widehat{f}_{0}}{\mathrm{D} v_{j}}=-2 \Re\left\{\boldsymbol{\lambda}^{T}\left(\frac{\partial \boldsymbol{\kappa}}{\partial v_{j}} \tilde{\boldsymbol{\Theta}}\right)\right\} \quad \text { for } j=1, \ldots, n
$$




\section{Results}

The acoustic model described in Section 2 has been implemented for the axisymmetric case in order to optimize the density layout of the absorbent material within the dissipative chamber. This section shows numerical results to illustrate the good performance of the proposed method. The designs obtained after optimization will be referred hereinafter as optimized designs, since it cannot be stated that they correspond to the global optima of their respective problems.

Given a muffler geometry, temperature distribution and Mach number at the inlet, computations are carried out with a view to maximize acoustic attenuation performance in the targeted frequency range. The TO method described in Section 3 is applied below to two different muffler configurations: a dissipative muffler with one chamber and perforated tube; and a hybrid muffler containing a dissipative chamber with perforated tube, and a reactive chamber with extended outlet tube. The effect of temperature gradients on the design of the noise control device will be included, as described in Section 5.1, as well as the simultaneous design of the density layout and the muffler's geometry given a constant temperature within it.

The computation times for the dissipative and hybrid geometries provided in Sections 5.2 and 5.3 refer to the use of an Intel@ Xeon $\AA$ CPU E5-2609 @ $2.40 \mathrm{GHz}$ with $16 \mathrm{~GB}$ RAM.

\subsection{Temperature distribution}

The temperature distribution within the muffler depends on the muffler configuration, as well as the engine load and speed, and affects the sound propagation properties of both the air and fibrous mediums. The effect of the absorbent material filling density on the temperature function is not included in this study, and therefore temperature can be expressed as a function of position $T=T(x, r)$ at all stages of optimization: a linear axial variation is assumed along the perforated section $T_{d u c t}(x)$, whereas temperatures at the inlet $T_{\text {in }}$ and outlet tubes $T_{\text {out }}$ are considered constant due to their short length with respect to the total length of the muffler, in accordance with reference [20]. 


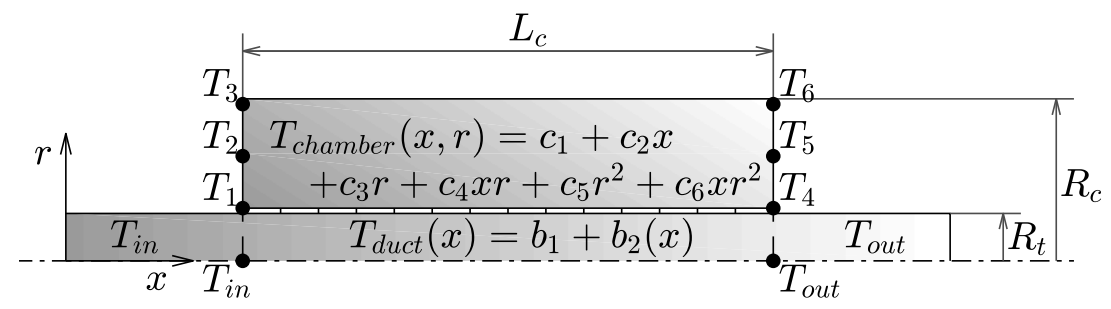

Figure 3: Axisymmetric temperature distribution studied.

Regarding the temperature function at the annular chamber, $T_{\text {chamber }}(x, r)$, a quadratic variation along the radial direction is added in order to approximate the logarithmic temperature function through a cylindrical domain. The example cases studied are shown in Table 2 [20].

\begin{tabular}{llllllllll}
\hline & $T_{\text {in }}$ & $T_{\text {out }}$ & $T_{1}$ & $T_{2}$ & $T_{3}$ & $T_{4}$ & $T_{5}$ & $T_{6}$ & $T_{\text {avg }}$ \\
\hline Case T-I & 25 & 25 & 25 & 25 & 25 & 25 & 25 & 25 & 25 \\
Case T-II & 300 & 200 & 300 & 235 & 200 & 200 & 135 & 100 & 181 \\
Case T-III & 181 & 181 & 181 & 181 & 181 & 181 & 181 & 181 & 181 \\
\hline
\end{tabular}

Table 2: Definition of the temperature field $\left({ }^{\circ} \mathrm{C}\right)$.

\subsection{Multi-frequency optimization of a dissipative muffler}

The configuration to optimize is shown in Fig. 4. The muffler consists of a single dissipative chamber, filled with Owens Corning texturized fibreglass roving [18] (see properties in Table 1). Its dimensions are: inner radius $R_{t}=0.0268 \mathrm{~m}$, outer radius $R_{c}=0.0886 \mathrm{~m}$, and chamber length $L_{c}=0.3$ $\mathrm{m}$. The perforated duct properties are: thickness $t_{p}=0.001 \mathrm{~m}$, porosity $\sigma=20 \%$, and orifice diameter $d_{h}=0.0035 \mathrm{~m}$.

$\Omega_{a}$ and $\Omega_{m}$ are meshed using 8-node quadrilateral elements, whose maximum length is set to $0.005 \mathrm{~m}$. 780 elements are obtained within $\Omega_{m}$ and therefore, 780 design variables are considered, with maximum and minimum element filling density values set to 50 and $250 \mathrm{~kg} / \mathrm{m}^{3}$ respectively. Initially, a homogeneous filling density of $120 \mathrm{~kg} / \mathrm{m}^{3}$ is assigned to every finite element of the chamber, using $0.8066 \mathrm{~kg}$ of absorbent material. This initial weight is defined as the maximum admissible weight $\left(W_{0}\right)$. Finally, the maximum change in each variable per iteration is restricted to $10 \%$ of the range. 


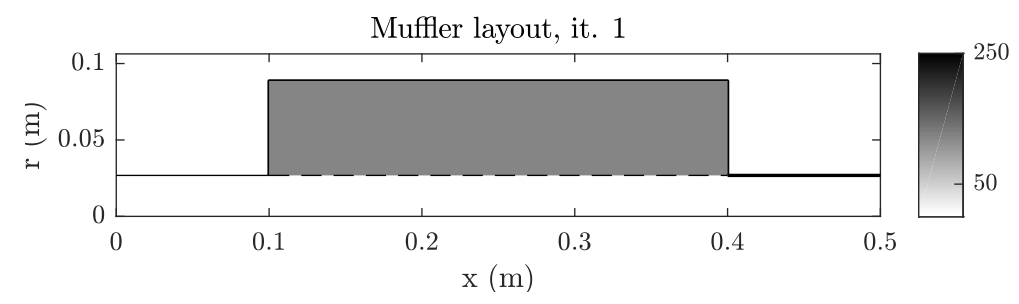

Figure 4: Initial design.

\section{Design 1. Cold condition. Low frequency range}

First, the TO process is carried out for the case T-I described in Table 2 (cold condition). Mach number at the inlet section is 0.05. The targeted frequency range where TL is to be improved is set to $[100-200] \mathrm{Hz}$. The acoustic analysis is performed by employing 4500 degrees of freedom, and the sensitivities of $f_{0}$ are obtained for each of the 101 frequencies of the Simpson's quadrature, with $\Delta \omega=5 \mathrm{~Hz}$. The MMA method is then used to optimize the material distribution by using gradient information. Each iteration takes 80 seconds to solve, using the aforementioned processor.

The stopping criterion is defined as the relative variation (in absolute value) in each design variable being below $1 \%$ of its range. Figure 5 shows the muffler design after meeting the stopping criterion at iteration 27.

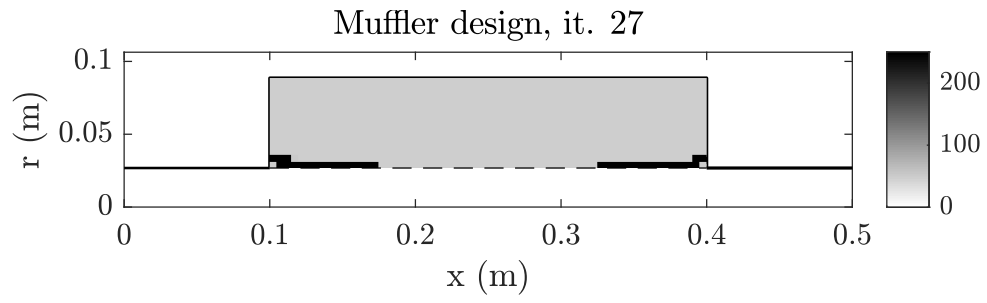

Figure 5: Optimized topology. Design 1.

As shown in Figure 5, the improved design has the minimum density associated with most of the elements of $\Omega_{m}$. Therefore the final design uses only $0.3656 \mathrm{~kg}$ of absorbent material. In accordance with Selamet et al. in [16], a slight improvement in TL at low frequencies can be achieved by using low filling density, even though this may result in a worse attenuation in the rest of the frequency range, as shown in Figure 7.

Even if this optimized muffler could be difficult or expensive to manufacture, the TO process yields basic ideas to construct a high-performance manufacturable muffler for these particular working conditions. 
Design 1a. Manufacturable muffler with $\rho_{b}=50 \mathrm{~kg} / \mathrm{m}^{3}$ and extended ducts

Design 1 shown in Figure 5 shows maximum density in two thin regions at both sides of the perforated surface. Although Design 1 is difficult to obtain by standard fabrication methods, it gives some guidelines in order to generate new designs. The increase in bulk density up to the maximum value next to the perforated duct tends to form acoustically independent subdomains, similar to an extended duct separating the central passage and the outer chamber. Therefore, it is suggested that Design 1 can be replaced by a muffler with homogeneous minimum filling density in the chamber and duct extensions (Design 1a). Figure 6 shows the easily manufacturable design with a homogeneous $\rho_{b}$ of $50 \mathrm{~kg} / \mathrm{m}^{3}$ and extended ducts of length $0.0075 \mathrm{~m}$ each, as suggested by Figure 5 .

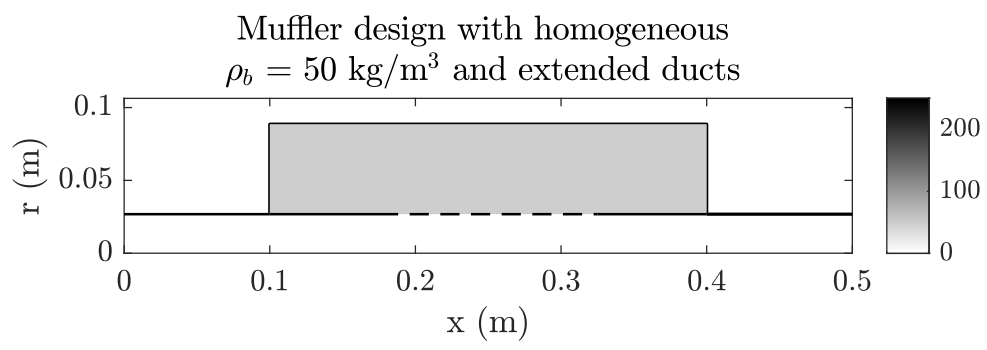

Figure 6: Manufacturable design. Design 1a.

Results discussion. Designs 1 and $1 a$

The attenuation performance of the optimized topology shown in Figure 5 (Design 1) and the manufacturable muffler shown in Figure 6 (Design 1a) are compared with the initial topology. As shown in Figure 7, the manufacturable design shows an improvement in terms of TL which is similar to the optimized design in the targeted frequency range, with respect to the initial design. In addition, both Designs 1 and 1a show worse attenuation with respect to the initial topology, for frequencies out of the attenuation range. The addition of the extended ducts does affect the TL prediction for higher frequencies: the manufacturable design shows better performance up to $1000 \mathrm{~Hz}$ with respect to the optimized design, while it provides worse attenuation in the range from 1000 to $3200 \mathrm{~Hz}$. 


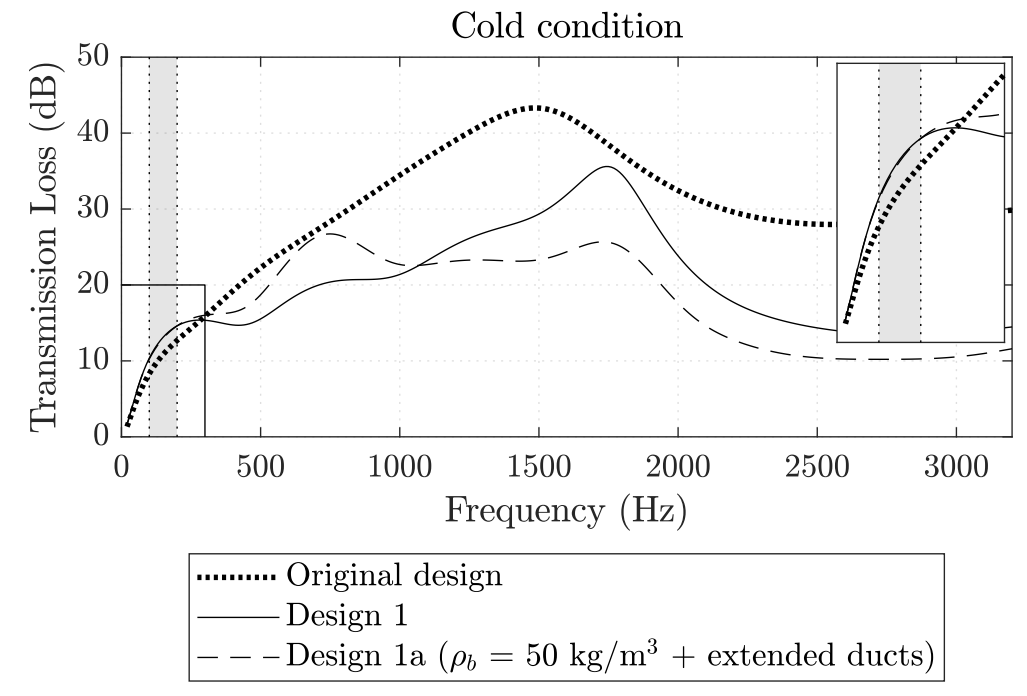

Figure 7: TL for the initial and optimized topologies (case 2 and 3).

Design 2. Cold condition. Mid frequency range

Next, the targeted frequency range is switched to [500 - 1000] Hz. The TO process is repeated for the same temperature case. Figure 8 shows an intermediate design of the optimization process, after 9 iterations. At this stage of the process, the design still includes many areas with intermediate values of density. However, the improved design shown in Figure 11 resembles a set of annular rings with the maximum and minimum filling densities. Similar configurations have been studied in reference [17].

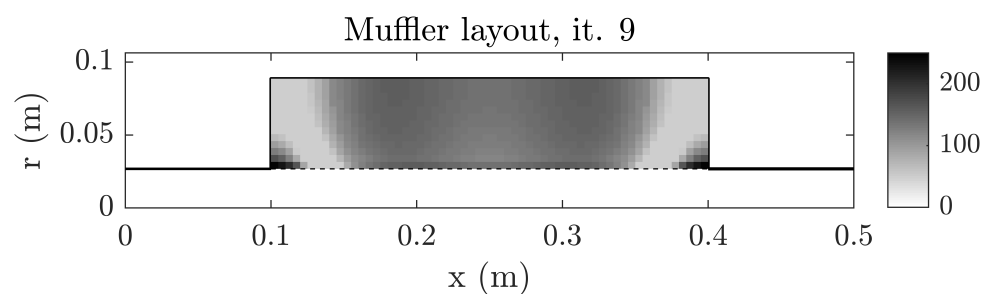

Figure 8: Optimized topology. Case 2 at iteration 9.

Figure 9 shows that $f_{0}$ improves at every iteration, from the starting value of $28.39 \mathrm{~dB}$ to $35.65 \mathrm{~dB}$ at iteration 43 . However, the value of the weight constraint $g_{1}$ oscillates until converging to a value near zero. This implies 
that the optimized design shown in Figure 11 (once optimization finishes) uses $0.8066 \mathrm{~kg}$ of material, as much as the initial one.

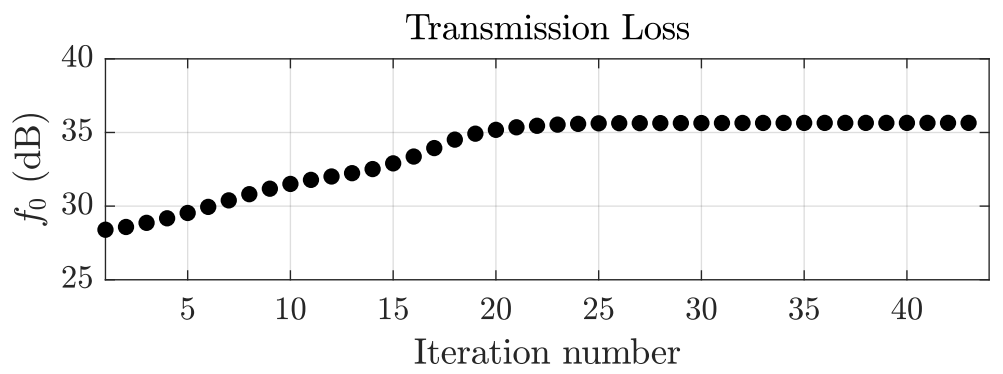

Figure 9: Case 2. Evolution of $f_{0}(\boldsymbol{v})$.

This suggests that better attenuation at mid frequencies could be obtained by the use of a higher amount of fibrous material, in accordance with reference [16].

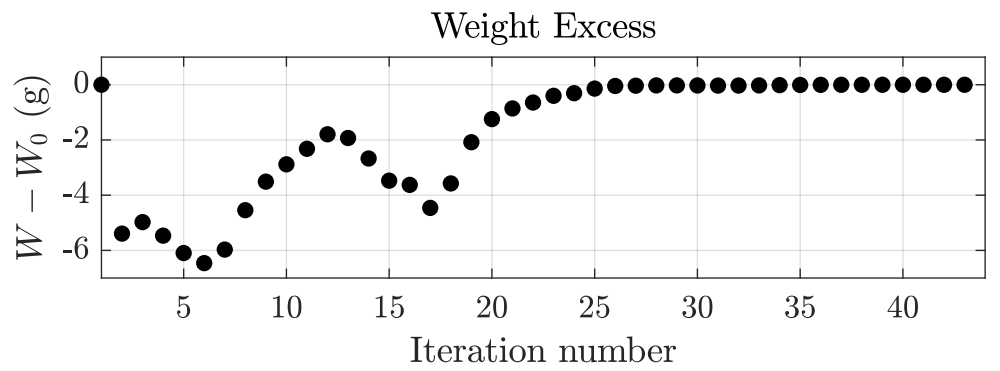

Figure 10: Case 2. Evolution of $g_{1}(\boldsymbol{v})$.

After 43 iterations, the stopping criterion is met. The resultant design, resembling a configuration with rings [17], is shown in Figure 11.

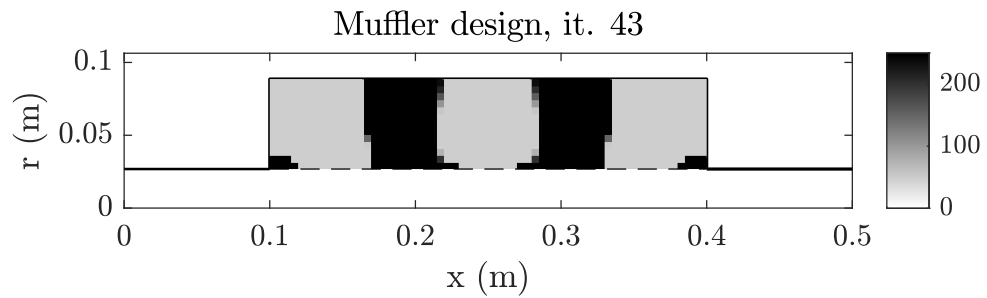

Figure 11: Optimized topology. Design 2. 
Design 2a. Manufacturable muffler with a ring design

Analogously to Case study 1, the optimized topology shown in Figure 11 is not easily manufacturable. Additionally, a ring configuration consisting of 5 rings of material with filling densities of 50 and $250 \mathrm{~kg} / \mathrm{m}^{3}$ is studied, as TO suggests. The rings are $0.065 \mathrm{~m}$ and $0.0525 \mathrm{~m}$ wide respectively, as shown in Figure 12, in order to maintain the amount of dissipative material used.

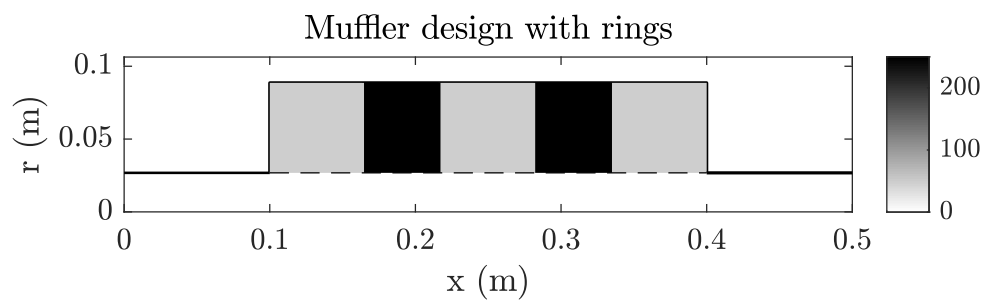

Figure 12: Manufacturable design. Design 2a.

Design 2b. Manufacturable muffler with a ring design and extended ducts

Design 2 shown in Figure 11 includes small regions of dissipative material with high density over both sides of the perforated surface, reducing the acoustic coupling between the subdomains $\Omega_{a}$ and $\Omega_{m}$ in these sections of the tube. Figure 8 shows that these areas tend to adopt the maximum bulk density faster than any other region in the chamber. As shown in the literature [34], the use of extended inlet/outlet ducts can help to increase attenuation at certain ranges of frequency. Therefore, an additional design including extended tubes with $0.02 \mathrm{~m}$ of length (equal to the axial length of 4 finite elements within $\Omega_{m}$ ) is studied. This design is sketched in Figure 13. The acoustic performance provided by Designs $2,2 \mathrm{a}$ and $2 \mathrm{~b}$ is shown in Figures 15 and 16, and results are discussed below in this section.

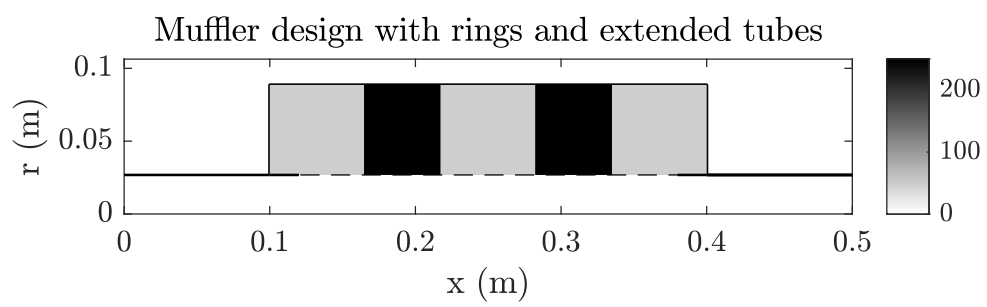

Figure 13: Manufacturable design. Design 2b. 
Case 3. Hot condition. Middle frequency range

The TL provided by a given muffler can differ substantially when considering thermal effects, and peaks in TL can shift to different frequencies $[20,22,40]$. Additionally, by considering silencers with large dimensions and fibre volume, high temperature and highly resistive fibres, it is likely that the topologies obtained by cold and hot TO could exhibit differences in some cases.

Next, the effect of temperature on the TO process will be studied, by considering the hot condition case T-II described in Table 2, while maintaining the frequency range of interest in $[500-1000] \mathrm{Hz}$. The initial density distribution is the one used in previous sections (see Fig. 4). The improved topology after stopping criterion is met at iteration 39 is plotted in Fig. 14.

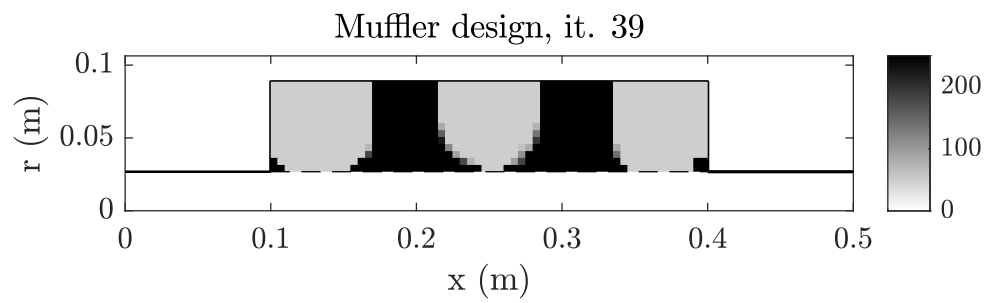

Figure 14: Optimized topology. Case 3.

As shown in Figure 14, by considering the high-temperature effect, a similar optimized topology to Design 2a (resultant of the cold TO) is obtained. It consists of 5 rings alternating minimum and maximum $\rho_{b}$. The final weight is again $0.8066 \mathrm{~kg}$.

Results discussion. Designs 2, 2a, 2b and 3

The initial design as well as Designs 2, 2a, 2b and 3 are evaluated at the cold temperature case T-I described in Table 2. The initial homogeneous topology of $120 \mathrm{~kg} / \mathrm{m}^{3}$ provides a mean TL in the targeted frequency range of $28.65 \mathrm{~dB}$. As it can be seen in Figure 15, the result of TO for the cold working condition (Design 2), shows good acosutic attenuation improvement in terms of TL (35.65 dB). Design 2a, consisting of 5 rings inferred from Design 2, provides a similar mean TL of $34.80 \mathrm{~dB}$. However, Design 2b with rings and extended ducts improves noise mitigation up to $37.02 \mathrm{~dB}$.

This suggests that the small regions with maximum $\rho_{b}$ that appear at both sides of the perforated surface have a physical meaning: the algorithm 
increases filling density in these small areas in order to reduce the acoustic coupling between the duct and the chamber in these regions of the perforated duct, similar to duct extensions. As shown in Figure 8, at iteration 9 of the TO, these small regions already show maximum $\rho_{b}$, whereas the rest of the finite elements within $\Omega_{m}$ are associated with intermediate filling densities. Therefore, removing the acoustic coupling at these regions by means of extended ducts increases attenuation in the problem under analysis. Finally, Design 3 shows worse attenuation than Design 2 at target frequencies (mean TL of $35.12 \mathrm{~dB}$ ). Nonetheless, the differences in material performance observed in the literature $[28,40]$ can justify the use of the complete model, which considers thermal effects.

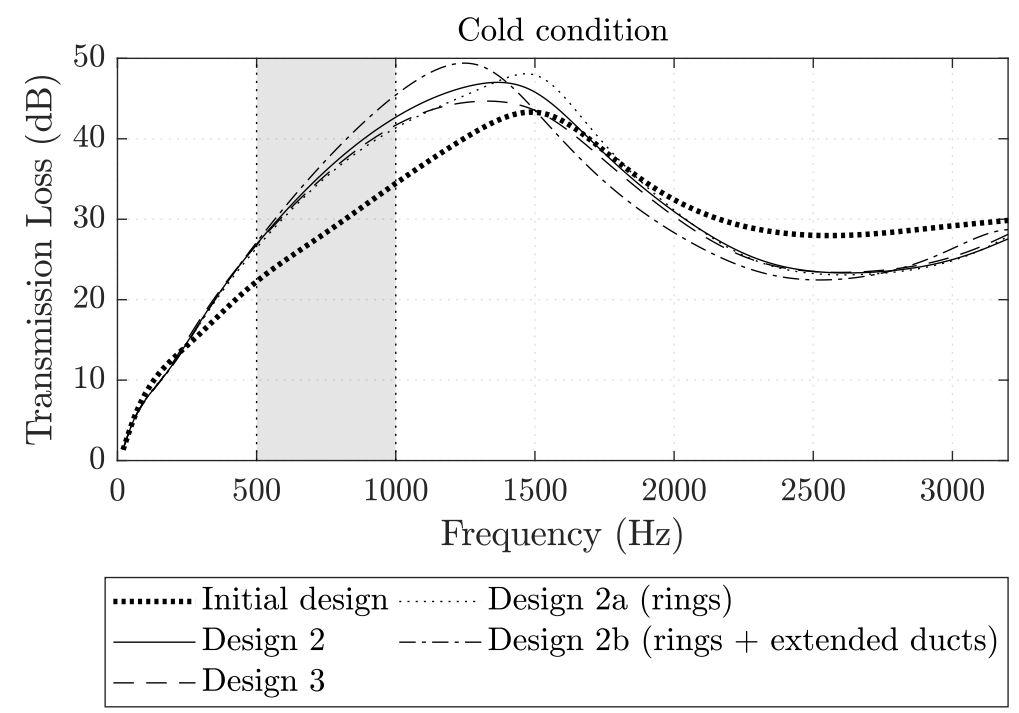

Figure 15: TL for the initial and optimized topologies (case 2 and 3).

On the other hand, the topologies obtained with the different working conditions are tested with the high temperature field T-II defined in Table 2. Results are shown in Figure 16. The initial topology with uniform $\rho_{b}$ of $120 \mathrm{~kg} / \mathrm{m}^{3}$ provides a mean TL at the targeted frequency range of $27.65 \mathrm{~dB}$. Design 2, resultant of the cold TO (case T-I of Table 2), provides a mean TL of $36.72 \mathrm{~dB}$ in the targeted range.

Design 3, obtained from the TO with the hot case T-II, provides a good improvement in TL in the targeted frequency range, up to $37.35 \mathrm{~dB}$. This proves that higher attenuation can be achieved with the same amount of 
material, depending on the working conditions. Figure 16 shows a peak in attenuation with $60 \mathrm{~dB}$ at around $1200 \mathrm{~Hz}$ for this last design.

Design 2a, consisting of rings, shows a mean TL of $35.30 \mathrm{~dB}$, which is worse than cases 2 and 3, but still better than the initial design. However, the addition of the extended ducts improves the mean TL up to $37.42 \mathrm{~dB}$, although their length is larger than the length suggested by Design 3 (see Figure 14).

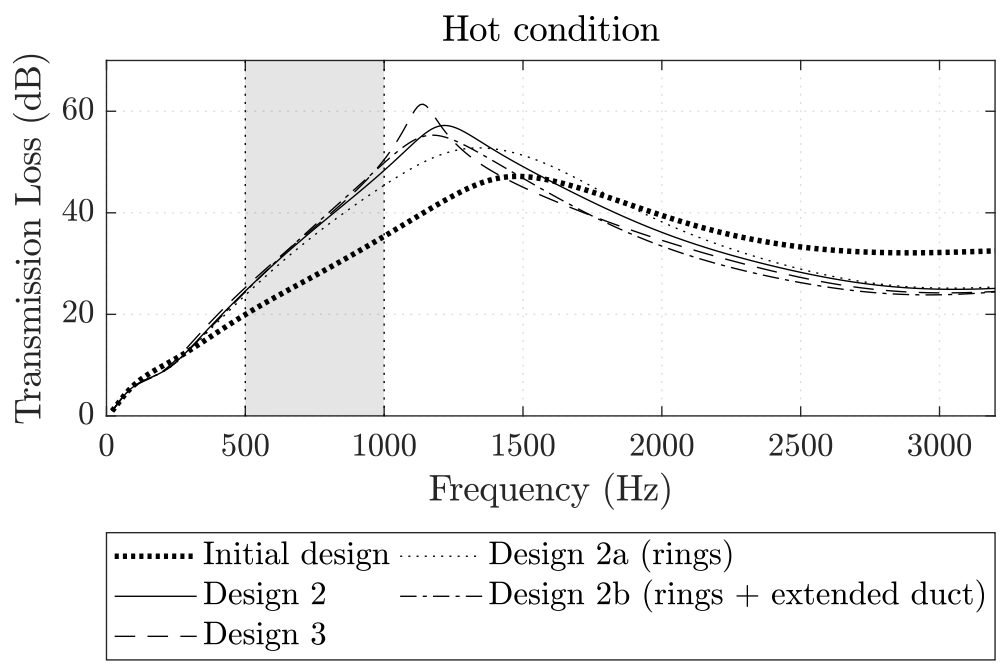

Figure 16: TL for the initial and optimized topologies (case 2 and 3).

For both cold and hot evaluations, all the optimized designs show worse TL than the initial design from around 1500 to $3200 \mathrm{~Hz}$, proving that optimization in one range of frequencies can lead in some cases to worse attenuation at other frequencies.

\subsection{Multi-frequency optimization of a hybrid muffler with multiple constraints}

Design 4. Broad frequency range

A hybrid muffler, which consists of a dissipative chamber and a reactive one with extended outlet duct, is considered in this section in order to maximize the attenuation in a wide range of frequencies. A baffle with $0.005 \mathrm{~m}$ in thickness is added between both chambers. In order to obtain potentially manufacturable designs, and taking into account the optimized topology obtained in previous cases, 5 annular rings with constant filling 
density are defined, each one containing several elements of the FE mesh, hence reducing the number of design variables.

The dimensions of these rings are also considered design variables $v_{j}$, as shown in Figure 17. The maximum and minimum values of each design variable can be checked in Table 3 . The sensitivities of $f_{0}$ with respect to these dimensions are also required at each iteration (see Appendix A.2 and Appendix A.3). Temperature gradients are neglected in this case for simplicity, and case T-III (hot muffler with constant temperature) detailed in Table 2 is considered. The targeted frequency range is [500-2000] Hz.

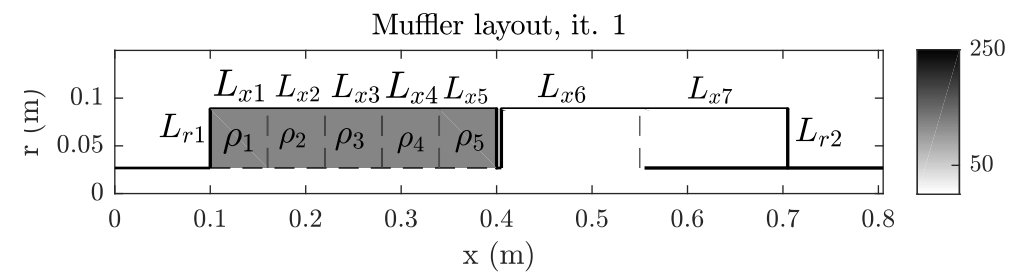

Figure 17: Initial design and design variables.

8146 nodes are employed, and each iteration (with 301 frequencies of analysis) takes 360 seconds by using the aforementioned processor.

Additional constraints are added in this case in order to keep the muffler under $1 \mathrm{~m}$ in length and both chambers with the same radii. The mean Mach number at the inlet is set to zero. The optimization stopped after 22 iterations producing the result shown in Figure 18.

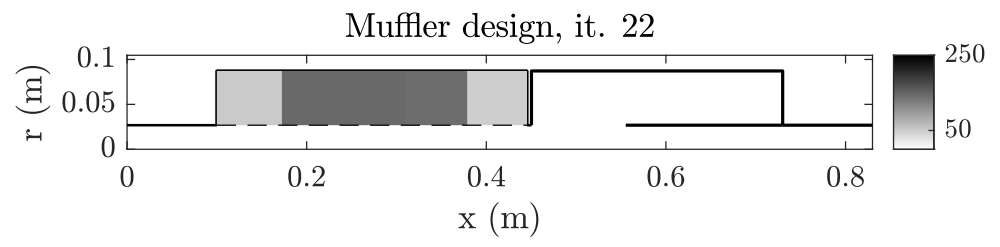

Figure 18: Optimized topology. Case 1.

Results discussion. Design 4

Figure 18 shows the optimized design. The left dissipative chamber contains two lateral rings with minimum $\rho_{b}$, and three central rings with around $150 \mathrm{~kg} / \mathrm{m}^{3}$. This chamber resembles the muffler configuration with two short lateral chambers and a central dissipative region, already studied in reference 
[41]. Also, an overall length increase of the dissipative chamber is observed, increasing the perforated surface length (and hence the area of acoustic coupling). The reactive chamber decreases in length, while containing a longer extended tube. Results are shown in detail in Table 3. The optimized design uses $0.8054 \mathrm{~kg}$ of absorbent material, and both additional constraints are satisfied (the muffler length is $0.838 \mathrm{~m}$ and both chambers have the same radius, $0.0872 \mathrm{~m})$.

\begin{tabular}{crrrr}
\hline$v_{j}$ & $v_{j}^{\text {min }}$ & $v_{j}^{\text {max }}$ & $v_{j}^{1}$ & $v_{j}^{\text {opt }}$ \\
\hline$\rho_{1}\left(\mathrm{~kg} / \mathrm{m}^{3}\right)$ & 50 & 250 & 120 & 51.07 \\
$\rho_{2}\left(\mathrm{~kg} / \mathrm{m}^{3}\right)$ & 50 & 250 & 120 & 145.81 \\
$\rho_{3}\left(\mathrm{~kg} / \mathrm{m}^{3}\right)$ & 50 & 250 & 120 & 148.27 \\
$\rho_{4}\left(\mathrm{~kg} / \mathrm{m}^{3}\right)$ & 50 & 250 & 120 & 141.17 \\
$\rho_{5}\left(\mathrm{~kg} / \mathrm{m}^{3}\right)$ & 50 & 250 & 120 & 50.00 \\
$L_{x 1}(\mathrm{~m})$ & 0.0420 & 0.7800 & 0.0600 & 0.0733 \\
$L_{x 2}(\mathrm{~m})$ & 0.0420 & 0.7800 & 0.0600 & 0.0686 \\
$L_{x 3}(\mathrm{~m})$ & 0.0420 & 0.7800 & 0.0600 & 0.0685 \\
$L_{x 4}(\mathrm{~m})$ & 0.0420 & 0.7800 & 0.0600 & 0.0685 \\
$L_{x 5}(\mathrm{~m})$ & 0.0420 & 0.7800 & 0.0600 & 0.0664 \\
$L_{r 1}(\mathrm{~m})$ & 0.0433 & 0.0803 & 0.0618 & 0.0604 \\
$L_{x 6}(\mathrm{~m})$ & 0.1050 & 0.1950 & 0.1500 & 0.1050 \\
$L_{x 7}(\mathrm{~m})$ & 0.1050 & 0.1950 & 0.1500 & 0.1747 \\
$L_{r 2}(\mathrm{~m})$ & 0.0433 & 0.0803 & 0.0618 & 0.0604 \\
\hline
\end{tabular}

Table 3: Optimization summary. Design parameter $v_{j}$, minimum $v_{j}^{\text {min }}$ and maximum $v_{j}^{\max }$ limits, initial $v_{j}^{1}$ and optimized $v_{j}^{\text {opt }}$ values. Case 4 .

The mean TL increase along the range of frequencies under study can be checked in Figure 19. The optimized design shows higher mean attenuation in the targeted frequency range $(63.84 \mathrm{~dB})$ than the initial design $(56.31 \mathrm{~dB})$, although it might be lower at some specific frequencies. Also, the peaks in TL shift to the left in Figure 19 as a result of the increase in the extended duct length. 


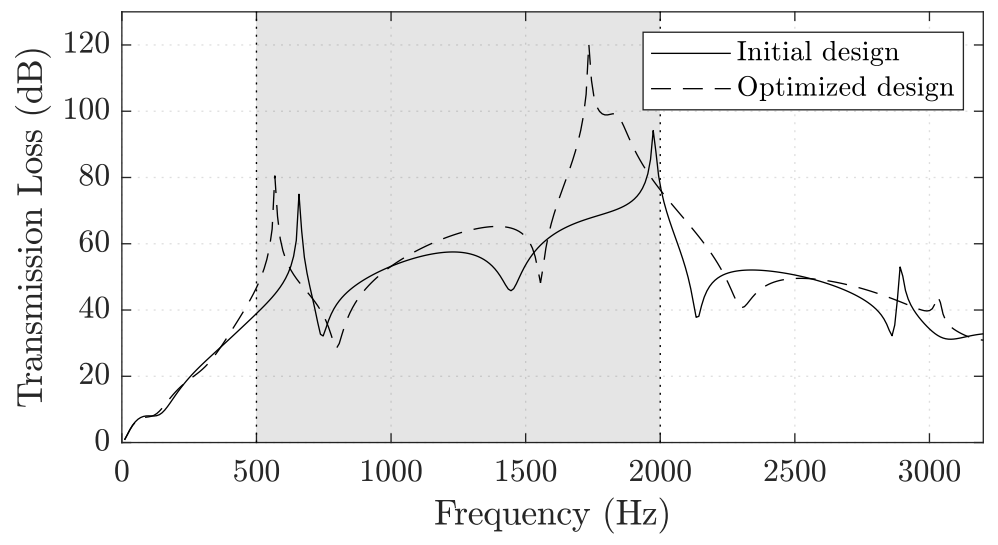

Figure 19: TL for the initial and optimized designs. Design 4.

\section{Conclusions}

This work presents a combination of geometry and topology optimization methods to effectively increase attenuation of dissipative and hybrid mufflers at certain frequency ranges. The evaluation of the TL is performed using a FE hybrid acoustic model valid for mufflers with heterogeneous absorbent material layout and perforated duct, in the presence of mean flow and temperature gradients. The optimization problem is solved by the use of the iterative algorithm MMA, which requires the gradient of both the objective function and the restrictions with respect to all design variables at each design step. The adjoint method, along with the analytical derivation of the global matrices with respect to each design variable, allows to efficiently calculate the sensitivities of the objective function.

For low frequencies, TO results in a reduction of the amount of dissipative material used in the dissipative chamber and a slight improvement in TL at target frequencies, which can worsen the acoustic behaviour at mid to high frequencies. Regarding the optimization at mid frequencies, the optimization scheme has been set up for two case studies with different thermal gradients, obtaining the optimized absorbent material layout in each case. Although little discrepancy in the optimized material distribution was observed, results showed the importance of considering temperature in the optimization process. The proposed methodology is able to provide a predesign for building manufacturable mufflers. These manufacturable designs are inferred from the topologies proposed by the optimization algorithm and keep most of the improvement in acoustic performance at target frequencies. This fact enables 
the proposed methodology to be a powerful tool for engineers and designers to build efficient mufflers for practical applications.

Finally, an optimization of a hybrid muffler has been carried out. The density layout obtained from the previous optimized dissipative mufflers has been combined with the geometry optimization of the reactive chamber and the size of the dissipative rings. The optimization is also carried out by using the MMA. Significant TL increase at target frequencies has been achieved in every case under study.

\section{Acknowledgements}

The authors gratefully acknowledge the financial support of Ministerio de Ciencia, Innovación y Universidades-Agencia Estatal de Investigación and the European Regional Development Fund (projects TRA2017-84701-R and DPI2017-89816-R), as well as Generalitat Valenciana (project Prometeo/2016/007).

\section{Conflict of interest}

On behalf of all authors, the corresponding author states that there is no conflict of interest.

\section{Replication of results}

The optimized fibre density layouts relating to Cases 1-3 shown in Figures 5, 11 and 14, are accessible in http://aim.upv.es/doc/SMO-TO-mufflersreplication-of-results.xlsx. Additionally, the TL prediction for Cases 1-4 shown in Figures 7, 15, 16 and 19 respectively, are provided in numerical

form in the same link. 
[1] M.L. Munjal, Acoustics of Ducts and Mufflers, John Wiley \& Sons, 2nd Edition (2014).

[2] R. Barbieri, N. Barbieri, Finite element acoustic simulation based shape optimization of a muffler, Applied Acoustics 67 (2006) 346-357.

[3] Zoutendijk G. Methods of feasible directions, Elsevier, Amsterdam (1960).

[4] Y.C. Chang, M.C. Chiu, Shape optimization of one-chamber perforated plug/non-plug mufflers by simulated annealing method, International Journal for Numerical Methods in Engineering 74 (2008) 1592-1620.

[5] K.F. De Lima, A. Lenzi, R. Barbieri, The study of reactive silencers by shape and parametric optimization techniques, Applied Acoustics 72 (2011) 142-150.

[6] O. Sigmund, K. Maute, Topology optimization approaches. Structural and Multidisciplinary Optimization 48 (2013) 1031-1055.

[7] M. Stolpe, K. Svanberg, An alternative interpolation scheme for minimum compliance optimization. Structural and Multidisciplinary Optimization 22 (2001) 116-124.

[8] J.W. Lee, Y.Y. Kim, Topology optimization of muffler internal partitions for improving acoustical attenuation performance, International Journal for Numerical Methods in Engineering 80 (2009) 455-477.

[9] E.L. Yedeg, E. Wadbro, and M. Berggren, Interior layout topology optimization of a reactive muffler. Structural and Multidisciplinary Optimization 53 (2016) 645-656.

[10] O. Sigmund, Morphology-based black and white filters for topology optimization. Structural and Multidisciplinary Optimization 33 (2007) 401-424.

[11] J.W. Lee, Optimal topology of reactive muffler achieving target transmission loss values: Design and experiment, Applied Acoustics 88 (2015) 104-113. 
[12] K. Svanberg, The method of moving asymptotes - a new method for structural optimization, International Journal for Numerical Methods in Engineering 24 (1987) 359-373.

[13] F.M. Azevedo, M.S. Moura, W.M. Vicente, R. Picelli, R. Pavanello, Topology optimization of reactive acoustic mufflers using a bi-directional evolutionary optimization method. Structural and Multidisciplinary Optimization 58 (2017) 2239-2252.

[14] G.H. Yoon, Acoustic topology optimization of fibrous material with Delany-Bazley empirical material formulation, Journal of Sound and Vibration 332 (2013) 1172-1187.

[15] J.S. Lee, P. Göransson, Y.Y. Kim, Topology optimization for threephase materials distribution in a dissipative expansion chamber by unified multiphase modeling approach, Computer Methods in Applied Mechanics and Engineering 287 (2015) 191-211.

[16] A. Selamet, M.B. Xu, I.J. Lee, N.T. Huff, Dissipative expansion chambers with two concentric layers of fibrous material, International Journal of Vehicle Noise and Vibration 1 (2005) 341-357.

[17] A. Selamet, M.B. Xu, I.J. Lee, N.T. Huff, Effect of voids on the acoustics of perforated dissipative mufflers, International Journal of Vehicle Noise and Vibration 2 (2006) 357-372.

[18] A. Selamet, I.J. Lee, N.T. Huff, Acoustic attenuation of hybrid mufflers, Journal of Sound and Vibration 262 (2003) 509-527.

[19] M. Chiu, Optimization design of hybrid mufflers on broadband frequencies using the genetic algorithm, Archives of Acoustics 36(2011) 795-822.

[20] F.D. Denia, E.M. Sánchez-Orgaz, J. Martínez-Casas, R. Kirby, Finite element based acoustic analysis of dissipative mufflers with high temperature and thermal-induced heterogeneity, Finite Element in Analysis and Design 101 (2015) 46-57.

[21] A.G. Antebas, F.D. Denia, A.M. Pedrosa, F.J. Fuenmayor, A finite element approach for the acoustic modelling of perforated dissipative mufflers with non-homogeneous properties, Mathematical and Computer Modelling 57 (2013) 1970-1978. 
[22] E.M. Sánchez-Orgaz, Advanced numerical techniques for the acoustic modelling of materials and noise control devices in the exhaust system of internal combustion engines, Ph. D. Thesis, Universitat Politècnica de València (2016).

[23] J.F. Allard, N. Atalla, Propagation of sound in porous media: Modelling sound absorbing materials (2009), Wiley, Chichester.

[24] M.E. Delany, E.N. Bazley, Acoustical properties of fibrous absorbent materials, Applied Acoustics 3 (1970) 105-116.

[25] R. Kirby, A. Cummings, Prediction of the bulk acoustic properties of fibrous materials at low frequencies, Applied Acoustics 56 (1999) 101125.

[26] D.R.A. Christie, Measurement of the acoustic properties of a soundabsorbing material at high temperatures, Journal of Sound and Vibration 46 (1976) 347-355.

[27] F.D. Denia, E.M. Sánchez-Orgaz, L. Baeza, R. Kirby, Point collocation scheme in mufflers with temperature gradient and mean flow, Journal of Computational and Applied Mathematics 291 (2016) 127-141.

[28] P.T. Williams, R. Kirby, C. Malecki, J. Hill, Measurement of the bulk acoustic properties of fibrous materials at high temperatures, Applied Acoustics 77 (2014) 29-36.

[29] A.D. Pierce, Wave equation for sound in fluids with unsteady inhomogeneous flow, Journal of the Acoustical Society of America 87 (1990) 2292-2299.

[30] O.C. Zienkiewicz, R.L. Taylor, J.Z. Zhu, The Finite Element Method: Its Basis and Fundamentals, Elsevier Butterworth-Heinemann, Burlington (2005).

[31] K.S. Peat, K.L. Rathi, A finite element analysis of the convected acoustic wave motion in dissipative mufflers, Journal of Sound and Vibration 184 (1995) 529-545.

[32] R. Kirby, F.D. Denia, Analytic mode matching for a circular dissipative muffler containing mean flow and a perforated pipe, Journal of the Acoustical Society of America 122 (2007) 3471-3482. 
[33] S.H. Lee, J.G. Ih, Empirical model of the acoustic impedance of a circular orifice in grazing mean flow, Journal of the Acoustical Society of America 114 (2003) 98-113.

[34] F.D. Denia, A. Selamet, F.J. Fuenmayor, R. Kirby, Acoustic attenuation performance of perforated dissipative mufflers with empty inlet/outlet extensions, Journal of Sound and Vibration 302 (2007) 1000-1017.

[35] K.U. Ingard, On the design of acoustic resonators, Journal of the Acoustical Society of America 25 (1953) 1037-1061.

[36] V.A. Fok, Doklady Akademii nauk, SSSR 31 (1941), in Russian. Alternatively, see S.N. Rschevkin, A course of lectures on the theory of sound, Pergamon, London, 1963.

[37] S.S. Rao, The Finite Element Method in Engineering, ButterworthHeinemann, 5th Edition (2011).

[38] K.E. Atkinson, An Introduction to Numerical Analysis. John Wiley \& Sons, 2nd Edition (1989).

[39] J.S. Jensen, Topology optimization. In: F. Romeo, M. Ruzzene. Wave Propagation in Linear and Nonlinear Periodic Media. CISM Courses and Lectures, vol 540. Springer, Vienna.

[40] R. Kirby, P.T. Williams, J. Hill, The effect of temperature on the acoustic performance of splitter silencers, 42nd International Congress and Exposition on Noise Control Engineering - INTERNOISE, 7 (2013) 5826-5833.

[41] F.D. Denia, A. Selamet, M.J. Martínez, A.J. Torregrosa, Hybrid mufflers with short lateral chambers: analytical, numerical and experimental studies, 13th International Congress on Sound and Vibration (ICSV 13), Vienna, 2006. 


\section{Appendix A. Differentiation of the global matrix $\kappa$ with respect to the design variables}

In this appendix, the expressions of each of the components of $\boldsymbol{\kappa}$ are differ-

entiated analytically with respect to each of the design variables considered in this study.

Appendix A.1. Calculation of $\partial \boldsymbol{\kappa} / \partial \rho_{b}$

Differentiating the global matrix $\boldsymbol{\kappa}$ in Eq. (54) with respect to the bulk density $\rho_{b}$ assigned to the elements $e=1, \ldots, N_{\rho}^{e}$, one obtains:

$$
\frac{\partial \boldsymbol{\kappa}}{\partial \rho_{b}}=\frac{\partial \mathbf{K}}{\partial \rho_{b}}+j \omega \frac{\partial \mathbf{C}}{\partial \rho_{b}}-\omega^{2} \frac{\partial \mathbf{M}}{\partial \rho_{b}},
$$

where, according to Eqs. (24), (25) and (33)-(38), the terms considered in order to build the global matrices are:

$$
\begin{aligned}
\frac{\partial \mathbf{K}_{m}}{\partial \rho_{b}} & =\sum_{e=1}^{N_{\rho}^{e}} \int_{\Omega_{\rho}^{e}} \frac{-\partial \rho_{m} / \partial \rho_{b}}{\rho_{b}^{2}}(\nabla \mathbf{N})^{T}(\nabla \mathbf{N}) \mathrm{d} \Omega \\
\frac{\partial \mathbf{M}_{m}}{\partial \rho_{b}} & =\sum_{e=1}^{N_{\rho}^{e}} \int_{\Omega_{\rho}^{e}}\left(-\frac{\partial \rho_{m} / \partial \rho_{b}}{\rho_{m}^{2} c_{m}^{2}}-2 \frac{\partial c_{m} / \partial \rho_{b}}{\rho_{m} c_{m}^{3}}\right) \mathbf{N}^{T} \mathbf{N} \mathrm{d} \Omega \\
\frac{\partial \mathbf{K}_{a a}^{Z_{p}}}{\partial \rho_{b}} & =\sum_{e=1}^{N_{\rho}^{e}} \int_{\Gamma_{\rho}^{e} \cap \Gamma_{p}}-\frac{\rho_{0}^{2} U_{m f} \partial \tilde{Z}_{p} / \partial \rho_{b}}{\tilde{Z}_{p}^{2}} \mathbf{N}^{T} \frac{\partial \mathbf{N}}{\partial x} \mathrm{~d} \Gamma \\
\frac{\partial \mathbf{K}_{a m}^{Z_{p}}}{\partial \rho_{b}} & =\sum_{e=1}^{N_{\rho}^{e}} \int_{\Gamma_{\rho}^{e} \cap \Gamma_{p}}-\frac{\rho_{0} \partial \tilde{Z}_{p} / \partial \rho_{b}}{\tilde{Z}_{p}^{2}} \mathbf{N}^{T} \mathbf{N} \mathrm{d} \Gamma \\
\frac{\partial \mathbf{C}_{a a}^{Z_{p}}}{\partial \rho_{b}} & =\sum_{e=1}^{N_{\rho}^{e}} \int_{\Gamma_{\rho}^{e} \cap \Gamma_{p}}-\frac{\rho_{0}^{2} \partial \tilde{Z}_{p} / \partial \rho_{b}}{\tilde{Z}_{p}^{2}} \mathbf{N}^{T} \mathbf{N} \mathrm{d} \Gamma \\
\frac{\partial \mathbf{C}_{m m}^{Z_{p}}}{\partial \rho_{b}} & =\sum_{e=1}^{N_{\rho}^{e}} \int_{\Gamma_{\rho}^{e} \cap \Gamma_{p}}-\frac{\partial \tilde{Z}_{p} / \partial \rho_{b}}{\tilde{Z}_{p}^{2}} \mathbf{N}^{T} \mathbf{N} \mathrm{d} \Gamma \\
\frac{\partial \mathbf{C}_{m a}^{Z_{p}}}{\partial \rho_{b}} & =\sum_{e=1}^{N_{\rho}^{e}} \int_{\Gamma_{\rho}^{e} \cap \Gamma_{p}}-\frac{\rho_{0} U_{m f} \partial \tilde{Z}_{p} / \partial \rho_{b}}{\tilde{Z}_{p}^{2}} \mathbf{N}^{T} \frac{\partial \mathbf{N}}{\partial x} \mathrm{~d} \Gamma \\
\frac{\partial \mathbf{M}_{m a}^{Z_{p}}}{\partial \rho_{b}} & =\sum_{e=1}^{N_{\rho}^{e}} \int_{\Gamma_{\rho}^{e} \cap \Gamma_{p}}-\frac{\rho_{0} \partial \tilde{Z}_{p} / \partial \rho_{b}}{\tilde{Z}_{p}^{2}} \mathbf{N}^{T} \mathbf{N} \mathrm{d} \Gamma .
\end{aligned}
$$


Using the equivalent acoustic properties detailed in Eqs. (5) and (6), the derivatives of $\rho_{m}$ and $c_{m}$ with respect to $\rho_{b}$ can be obtained as:

$$
\begin{aligned}
\frac{\partial \rho_{m}}{\partial \rho_{b}}(\mathbf{x}) & =\frac{1}{c_{m}(\mathbf{x})^{2}}\left(\frac{\partial Z_{m}}{\partial \rho_{b}}(\mathbf{x}) c_{m}(\mathbf{x})-Z_{m}(\mathbf{x}) \frac{\partial c_{m}}{\partial \rho_{b}}(\mathbf{x})\right) \\
\frac{\partial c_{m}}{\partial \rho_{b}}(\mathbf{x}) & =-\frac{1}{k_{m}(\mathbf{x})^{2}} \frac{\partial k_{m}}{\partial \rho_{b}}(\mathbf{x})
\end{aligned}
$$

whereas according to Eqs. (3) and (4), it can be obtained:

$$
\begin{aligned}
& \frac{\partial Z_{m}}{\partial \rho_{b}}(\mathbf{x})=Z_{0}(\mathbf{x})\left(1+a_{5} a_{6} \xi(\mathbf{x})^{a_{6}-1}-j a_{7} a_{8} \xi(\mathbf{x})^{a_{8}-1}\right) \frac{\partial \xi}{\partial \rho_{b}}(\mathbf{x}) \\
& \frac{\partial k_{m}}{\partial \rho_{b}}(\mathbf{x})=k_{0}(\mathbf{x})\left(1+a_{3} a_{4} \xi(\mathbf{x})^{a_{4}-1}-j a_{1} a_{2} \xi(\mathbf{x})^{a_{2}-1}\right) \frac{\partial \xi}{\partial \rho_{b}}(\mathbf{x}) .
\end{aligned}
$$

Taking into account the thermal effects described in Christie's power law recalled in Eq. (2), the derivative of the frequency parameter with respect to $\rho_{b}$ is

$$
\frac{\partial \xi}{\partial \rho_{b}}(\mathbf{x})=\frac{-A_{1} A_{2} \rho_{b}(\mathbf{x})^{A_{2}-1} \rho_{0}(\mathbf{x}) f}{R^{2}(\mathbf{x})}\left(\frac{T(\mathbf{x})+273.15}{T_{0}+273.15}\right)^{0.6}
$$

On the other hand, differentiating Eq. (40), $\partial \tilde{Z}_{p} / \partial \rho_{b}$ can be obtained as:

$$
\frac{\partial \tilde{Z}_{p}(x)}{\partial \rho_{b}}=Z_{0}(x) \frac{j 0.425 k_{0}(x) d_{h} / \rho_{0}(x) F(\sigma)}{\sigma} \frac{\partial \rho_{m}}{\partial \rho_{b}}(\mathbf{x}) .
$$

Appendix A.2. Calculation of $\partial \boldsymbol{\kappa} / \partial L_{x}$

In Section 5.3, rings of absorbent material are defined as areas with constant $\rho_{b}$, and the dimensions of these are also subject to modification. Cartesian element grids are implemented within each ring. For the elements within a certain ring, $e=1, \ldots, N_{m}^{e}$, and the elements within the corresponding duct zone underneath, $e=1, \ldots, N_{a}^{e}$, the velocity field at the element integration points due to the modification of the ring length $L_{x}$ must be taken into 
account during the computation of the terms listed below:

$$
\begin{aligned}
& \frac{\partial \mathbf{K}_{a}}{\partial L_{x}}=\sum_{e=1}^{N_{a}^{e}} \int_{\Omega_{a}^{e}} \rho_{0}\left(\left(\frac{\partial(\nabla \mathbf{N})^{T}}{\partial L_{x}} \mathbf{M}(\nabla \mathbf{N})+(\nabla \mathbf{N})^{T} \mathbf{M} \frac{\partial(\nabla \mathbf{N})}{\partial L_{x}}\right) \mathrm{d} \Omega\right. \\
& \left.+(\nabla \mathbf{N})^{T} \mathbf{M}(\nabla \mathbf{N}) \frac{\partial(\mathrm{d} \Omega)}{\partial L_{x}}\right), \\
& \frac{\partial \mathbf{M}_{a}}{\partial L_{x}}=\sum_{e=1}^{N_{a}^{e}} \int_{\Omega_{a}^{e}} \frac{\rho_{0} \mathbf{N}^{T} \mathbf{N}}{c_{0}^{2}} \frac{\partial(\mathrm{d} \Omega)}{\partial L_{x}}, \\
& \frac{\partial \mathbf{K}_{m}}{\partial L_{x}}=\sum_{e=1}^{N_{m}^{e}} \int_{\Omega_{m}^{e}} \frac{1}{\rho_{m}}\left(\left(\frac{\partial(\nabla \mathbf{N})^{T}}{\partial L_{x}}(\nabla \mathbf{N})+(\nabla \mathbf{N})^{T} \frac{\partial(\nabla \mathbf{N})}{\partial L_{x}}\right) \mathrm{d} \Omega\right. \\
& \left.+(\nabla \mathbf{N})^{T}(\nabla \mathbf{N}) \frac{\partial(\mathrm{d} \Omega)}{\partial L_{x}}\right), \\
& \frac{\partial \mathbf{M}_{m}}{\partial L_{x}}=\sum_{e=1}^{N_{m}^{e}} \int_{\Omega_{m}^{e}} \frac{\mathbf{N}^{T} \mathbf{N}}{\rho_{m} c_{m}^{2}} \frac{\partial(\mathrm{d} \Omega)}{\partial L_{x}} \\
& \frac{\partial \mathbf{K}_{a a}^{Z_{p}}}{\partial L_{x}}=\sum_{e=1}^{N_{a}^{e}} \int_{\Gamma_{a}^{e} \cap \Gamma_{p}} \frac{\rho_{0}^{2} U_{m f} \mathbf{N}^{T}}{\tilde{Z}_{p}}\left(\frac{\partial(\partial \mathbf{N} / \partial x)}{\partial L_{x}} \mathrm{~d} \Gamma+\frac{\partial \mathbf{N}}{\partial x} \frac{\partial(\mathrm{d} \Gamma)}{\partial L_{x}}\right), \\
& \frac{\partial \mathbf{K}_{a m}^{Z_{p}}}{\partial L_{x}}=\sum_{e=1}^{N_{a}^{e}} \int_{\Gamma_{a}^{e} \cap \Gamma_{p}} \frac{\rho_{0} \mathbf{N}^{T} \mathbf{N}}{\tilde{Z}_{p}} \frac{\partial(\mathrm{d} \Gamma)}{\partial L_{x}}, \\
& \frac{\partial \mathbf{C}_{a a}^{Z_{p}}}{\partial L_{x}}=\sum_{e=1}^{N_{a}^{e}} \int_{\Gamma_{a}^{e} \cap \Gamma_{p}} \frac{\rho_{0}^{2} \mathbf{N}^{T} \mathbf{N}}{\tilde{Z}_{p}} \frac{\partial(\mathrm{d} \Gamma)}{\partial L_{x}}, \\
& \frac{\partial \mathbf{C}_{m m}^{Z_{p}}}{\partial L_{x}}=\sum_{e=1}^{N_{m}^{e}} \int_{\Gamma_{m}^{e} \cap \Gamma_{p}} \frac{\mathbf{N}^{T} \mathbf{N}}{\tilde{Z}_{p}} \frac{\partial(\mathrm{d} \Gamma)}{\partial L_{x}}, \\
& \frac{\partial \mathbf{C}_{m a}^{Z_{p}}}{\partial L_{x}}=\sum_{e=1}^{N_{m}^{e}} \int_{\Gamma_{m}^{e} \cap \Gamma_{p}} \frac{\rho_{0} U_{m f} \mathbf{N}^{T}}{\tilde{Z}_{p}}\left(\frac{\partial(\partial \mathbf{N} / \partial x)}{\partial L_{x}} \mathrm{~d} \Gamma+\frac{\partial \mathbf{N}}{\partial x} \frac{\partial(\mathrm{d} \Gamma)}{\partial L_{x}}\right) \\
& \frac{\partial \mathbf{M}_{m a}^{Z_{p}}}{\partial L_{x}}=\sum_{e=1}^{N_{m}^{e}} \int_{\Gamma_{m}^{e} \cap \Gamma_{p}} \frac{\rho_{0} \mathbf{N}^{T} \mathbf{N}}{\tilde{Z}_{p}} \frac{\partial(\mathrm{d} \Gamma)}{\partial L_{x}} .
\end{aligned}
$$

Appendix A.3. Calculation of $\partial \boldsymbol{\kappa} / \partial L_{r}$

In order to compute the derivative of the chamber terms with respect to the chamber dimension $L_{r}=R_{c}-R_{t}$ (see Figures 3 and 17), the velocity 
field generated by $\partial L_{r}$ must be taken into account, but also the axisymmetric integration effect must be considered when computing $\partial(\mathrm{d} \Omega) / \partial L_{r}$. The terms are given by the expressions:

$$
\begin{aligned}
\frac{\partial \mathbf{K}_{m}}{\partial L_{r}} & =\sum_{e=1}^{N_{m}^{e}} \int_{\Omega_{m}^{e}} \frac{1}{\rho_{m}}\left(\left(\frac{\partial(\nabla \mathbf{N})^{T}}{\partial L_{r}}(\nabla \mathbf{N})+(\nabla \mathbf{N})^{T} \frac{\partial(\nabla \mathbf{N})}{\partial L_{r}}\right) \mathrm{d} \Omega\right. \\
& \left.+(\nabla \mathbf{N})^{T}(\nabla \mathbf{N}) \frac{\partial(\mathrm{d} \Omega)}{\partial L_{r}}\right), \\
\frac{\partial \mathbf{M}_{m}}{\partial L_{r}} & =\sum_{e=1}^{N_{m}^{e}} \int_{\Omega_{m}^{e}} \frac{\mathbf{N}^{T} \mathbf{N}}{\rho_{m} c_{m}^{2}} \frac{\partial(\mathrm{d} \Omega)}{\partial L_{r}} .
\end{aligned}
$$

